\title{
1 \\ 2 \\ Dissecting the co-transcriptome landscape of plants and microbiota members
}

3 Tatsuya Nobori ${ }^{3,4}$, Yu $\mathrm{Cao}^{3}$, Frederickson Entila ${ }^{3}$, Eik Dahms ${ }^{3}$, Yayoi Tsuda ${ }^{1,2,3}$,

4 Ruben Garrido-Oter ${ }^{3,5}$, Kenichi Tsuda ${ }^{1,2,3^{*}}$

$5{ }^{1}$ Key Laboratory of Agricultural Microbiology, College of Plant Science and

6 Technology, Huazhong Agricultural University, Wuhan 430070, China.

$7{ }^{2}$ The Provincial Key Lab of Plant Pathology of Hubei Province, Huazhong

8 Agricultural University, Wuhan, China

9 3epartment of Plant Microbe Interactions, Max Planck Institute for Plant

10 Breeding Research, Carl-von-Linne-Weg 10, Cologne 50829, Germany.

$11{ }^{4}$ Salk Institute for Biological Studies, La Jolla, CA 92037, USA.

$12{ }^{5}$ Cluster of Excellence on Plant Sciences, 40225 Düsseldorf, Germany.

13 *Corresponding author. Email: tsuda@mail.hzau.edu.cn 


\section{Abstract}

15 Interactions between plants and each neighboring microbial species are

16 fundamental building blocks that collectively determine the structure and function

17 of the plant microbiota, but the molecular basis of such interactions is poorly

18 characterized. Here, we monocolonized Arabidopsis leaves with nine

19 plant-associated bacteria from all major phyla of the plant microbiota and profiled

20 co-transcriptomes of plants and bacteria. These strains elicited quantitatively

21 different plant transcriptional responses including typical pattern-triggered

22 immunity responses. Genes of non-pathogenic bacteria involved in general

23 metabolism and energy production were commonly suppressed in planta in

24 contrast to a virulent pathogen. Various nutrient acquisition pathways that are

25 frequently encoded in the genomes of plant-associated bacteria were induced in

26 planta in a strain-specific manner, shedding light on bacterial adaptation to the

27 plant environment and identifying a potential driving force of niche separation.

28 Integrative analyses of plant and bacterial transcriptomes suggested that the

29 transcriptional reprogramming of plants is largely uncoupled from that of bacteria

30 at an early stage of interactions. This study provides insights into how plants

31 discriminate among bacterial strains and sets the foundation for in-depth

32 mechanistic dissection of plant-microbiota interactions. 


\section{Introduction}

34 In nature, plants can reproducibly assemble bacterial communities with 35 well-defined taxonomic structures (the plant microbiota) (Hacquard et al., 2015),

36 which can be harnessed for plant health and survival (Carrión et al., 2019; Durán

37 et al., 2018; Kwak et al., 2018). How plants discriminate among various bacterial

38 strains and establish strain-specific associations in a community context remains

39 an open question in basic plant microbiota research and is key to facilitate the

40 application of microbiota-based strategies to improve plant health in agricultural

41 settings. Answering this question requires a comprehensive and unified

42 understanding of plant and bacterial responses during their interactions.

43 Plant responses to microorganisms are controlled by the plant innate immune 44 system, which contributes to the assembly and maintenance of healthy bacterial 45 communities (Chen et al., 2020; Lebeis et al., 2015). A crucial part of the plant 46 immune system is the perception of environmental microbes using cell surface 47 receptors that detect conserved microbial epitopes, termed microbe-associated 48 molecular patterns (MAMPs) (Boller and Felix, 2009). Recognition of MAMPs 49 triggers defense responses collectively called pattern-triggered immunity (PTI), 50 which can inhibit pathogen growth (Jones and Dangl, 2006). MAMPs such as the

51 bacterial flagellin peptide flg22 are widely conserved in non-pathogenic bacteria

52 as well as pathogenic bacteria (Hacquard et al., 2017), and some

53 non-pathogenic Proteobacteria strains were shown to trigger defense responses

54 in plant leaves likely via PTI pathways (Vogel et al., 2016). On the other hand,

55 diverse microbiota members can suppress PTI triggered by flg22 in roots

56 (Garrido-Oter et al., 2018; Teixeira et al., 2021; Yu et al., 2019), which can

57 facilitate colonization by the root microbiota (Teixeira et al., 2021; Yu et al., 2019).

58 Thus, PTI activation by divergent MAMPs and subsequent PTI modulation by 59 plant-associated bacteria might steer plant responses in a bacterial 60 strain-specific manner, contributing to microbiota assembly in roots. In leaves, 
61 however, our current understanding of plant responses to individual microbiota

62 members is largely limited to a specific phylum of bacteria, Proteobacteria, and

63 little is known about how plants respond to bacterial strains belonging to other

64 major phyla such as Actinobacteria, Bacteroidetes, and Firmicutes.

65 When colonized densely and heterogeneously by various bacterial species,

66 plants might not be able to tailor their responses to individual bacterial strains.

67 Yet, it might be possible that different plant-associated bacterial species respond

68 differently to the same microenvironments created by plants. If so, analyzing

69 plant responses alone does not wholly explain bacterial responses during

70 interactions with hosts. The explanation requires directly interrogating bacterial

71 responses in planta at the genome-wide scale. In planta bacterial omics

72 approaches, such as transcriptomics, are powerful in understanding bacterial

73 gene functions in the plant microbiome and how plants influence bacterial

74 activities (Levy et al., 2018). To date, however, there is a handful of in planta

75 bacterial transcriptome studies, all focusing on pathogenic Proteobacteria strains

76 (Chapelle et al., 2015; Lovelace et al., 2018; Nobori et al., 2018, 2020; Yu et al.,

77 2013, 2014). It is, therefore, unknown whether plant-associated bacteria have

78 any common or phylum-specific signatures in the usage of their genomes and

79 what kind of functions are important for their non-pathogenic and sometimes

80 beneficial traits in plants. Furthermore, to our knowledge, no study has analyzed

81 plant and bacterial transcriptome responses at the same time, limiting our ability

82 to build hypotheses about the molecular dialogue between plants and microbiota

83 members.

84 Here, in monoassociation conditions, we co-profiled the transcriptomes of the

85 model plant Arabidopsis thaliana and various bacterial strains isolated from

86 healthy (asymptomatic) plants in nature (hereafter commensal strains),

87 representing all major phyla of the plant microbiota residing in leaves.

88 Commensal strains commonly induced PTI responses in plants, but these

89 differed in intensity. We found examples of both common and strain-specific 
90 regulation of commensal gene expression in plants. Bacterial genes enriched in

91 plant-associated strains tended to be induced in planta. These included genes

92 involved in sulfur, nitrogen, and carbon transport and metabolism, which were

93 induced in planta in a strain-specific manner. This suggests that nutrient status

94 differs for different strains in plants, which may affect bacterial fitness and niche

95 separation. We also observed that plants could elicit different transcriptional

96 responses from different bacterial strains without tailoring their own

97 transcriptional reprogramming. This study provides a framework for dissecting

98 plant-microbiota interactions at the strain level using co-transcriptomics and

99 unravels diverse modes of interactions between plants and commensal bacteria.

\section{Results}

101 Co-transcriptome analysis of plants and plant microbiota 102 members

103 We developed a pipeline to simultaneously investigate the transcriptomes of both

104 plants and bacteria during plant colonization with a single bacterial strain. We

105 monocolonized $A$. thaliana wild-type Col-0 leaves with individual commensal

106 strains by hand-infiltration and profiled transcriptomes of plants and bacteria at 6

107 hours post-inoculation (hpi) by RNA-seq (Fig. 1A). For in planta bacterial

108 RNA-seq, we used a previously developed method with some modifications (see

109 Methods). Briefly, bacterial cells are isolated from plant leaves before extracting

110 RNA, followed by rRNA depletion and RNA-seq (Nobori et al., 2018). For plant

111 and bacterial RNA-seq, respectively, 18 and nine commensal strains were

112 selected covering all major phyla of the plant microbiota (Fig. 1B and Table 1).

113 Three biological replicates from independent experiments were taken for each

114 condition. We used the same strain IDs as in the original study where these

115 bacterial strains were isolated from wild $A$. thaliana plants (leaves and roots) or 
116 soil (Bai et al., 2015). A strain ID indicates the original compartment from which

117 the strain was isolated, but many root/soil isolates can also colonize the shoot,

118 indicating extensive niche overlap (Bai et al., 2015).

119 Nine commensal strains were used for co-transcriptome analysis (Fig. 1C).

120 These strains could colonize to various degrees in the leaf endosphere when

121 inoculated on the leaf surface (Fig. S1). For plants, we compared gene

122 expression changes between bacteria-inoculated plants and water-inoculated

123 plants (Fig. 1C, left). For bacteria, we compared expression changes between in

124 planta and in vitro (rich media) conditions (Fig. 1C, right; Fig. S2A). We also

125 included previously generated in plantalin vitro transcriptome data of the virulent

126 pathogen Pseudomonas syringae pv. tomato DC3000 (Pto) and its avirulent

127 mutant D36E (36 type III effectors are depleted) (Nobori et al., 2018) in the

128 analysis. To directly compare bacterial gene expression patterns among

129 phylogenetically diverse bacterial strains, genes of different strains were grouped

130 based on sequence homology, resulting in 6,823 orthologous groups (OGs) (Fig.

131 S2B). Of these OGs, only 454 OGs were shared among all strains (Data S1),

132 indicating that the commensal strains used in this study possess highly diverse

133 gene sets. Principal component analysis using the shared OGs showed that all

134 commensal strains except for Leaf1, showed relatively similar gene expression

135 patterns both in planta and under in vitro conditions (Fig. 1D). Interestingly, while

136 the gene expression pattern of the avirulent pathogen D36E in planta was similar

137 to those of commensals, the virulent Pto showed a distinct pattern, i.e., Pto in

138 planta resembled commensals in vitro (Fig. 1D). Thus, the commensal strains

139 used in this study have highly different genomes, but in planta expression of

140 shared genes is similar to each other and highly distinct from that of a virulent

141 pathogen. 
A

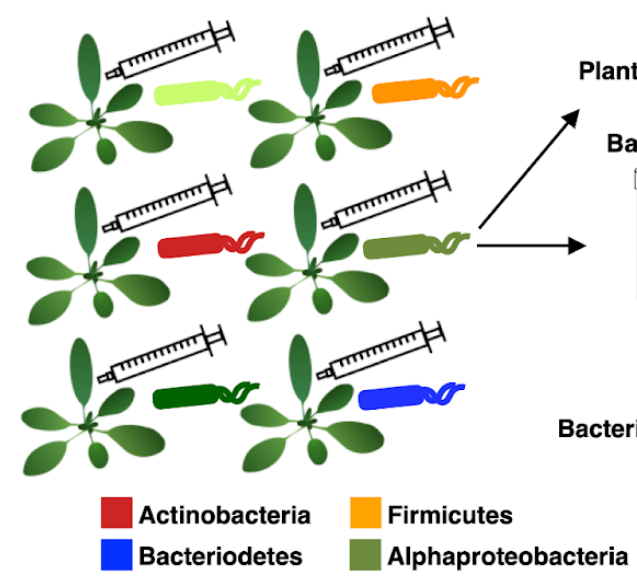

B

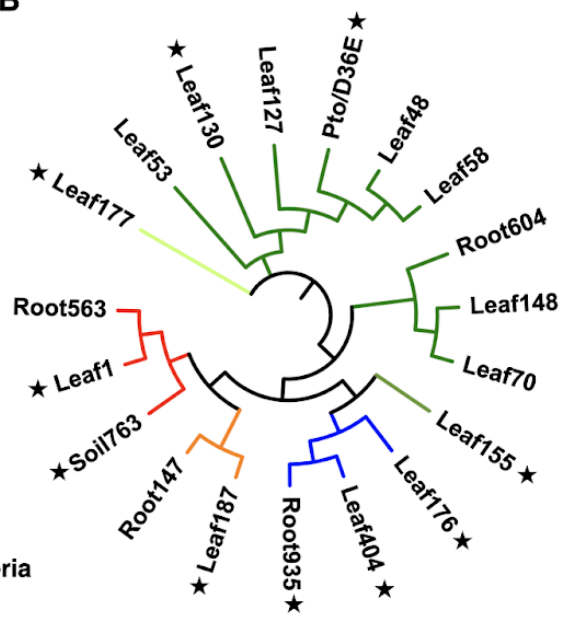

\section{Bacteria}

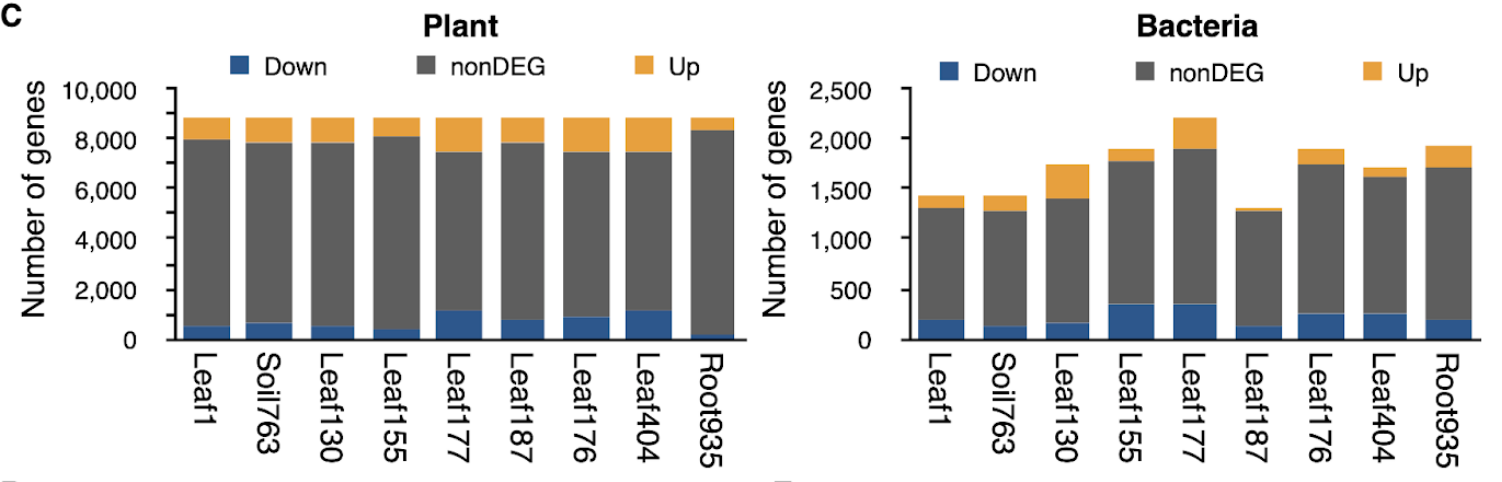

D

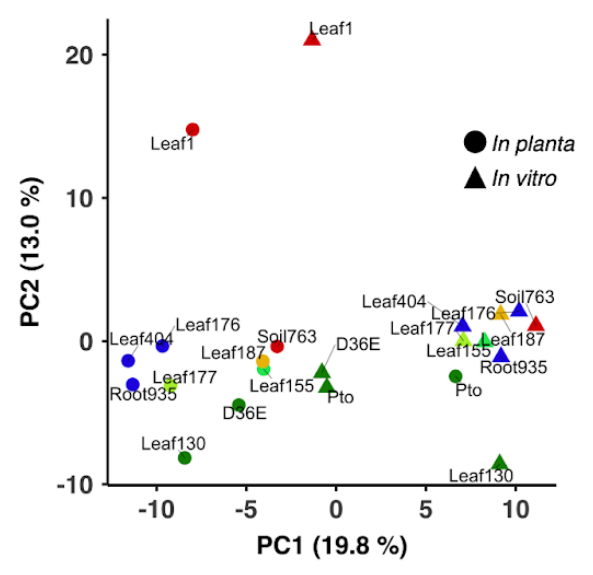

E

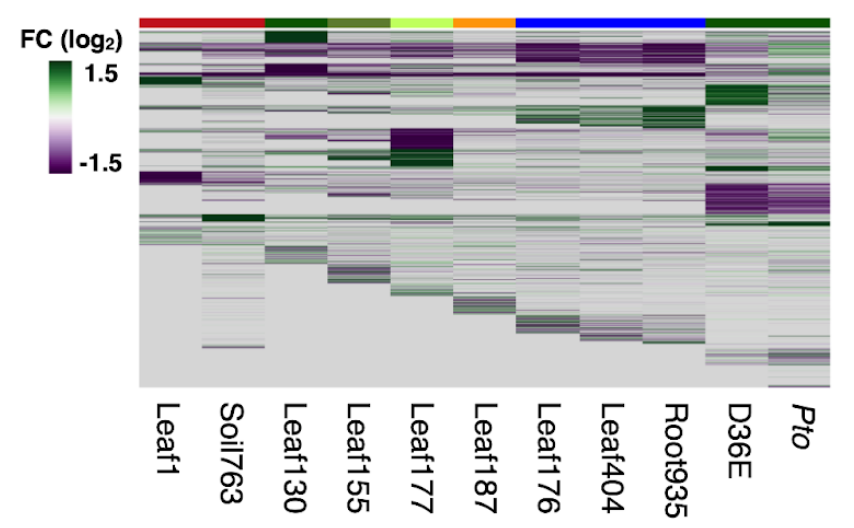

143 Fig. 1: Co-transcriptomics of plants and bacteria (A) Experimental scheme.

144 Individual bacterial strains were syringe-infiltrated into leaves of $A$. thaliana.

145 Leaves were sampled at $6 \mathrm{~h}$ post-inoculation. Total RNA was extracted for plant

146 RNA-seq. For bacterial RNA-seq, bacterial cells were isolated from plant leaves

147 before extracting RNA using a method previously reported (Nobori et al., 2018).

148 (B) Bacterial strains used in this study. Stars indicate the strains used for 149 co-transcriptome analysis. Detailed taxonomic information is shown in Table 1.

150 (C) The number of genes differentially regulated $\left(\left|\log _{2} \mathrm{FC}\right|>1\right.$; FDR $<0.01$; 151 two-tailed Student's t test followed by Storey's q-value) in plant or bacterial 
152 RNA-seq. Plant: bacteria-inoculated vs. water-inoculated. Bacteria: in planta $6 \mathrm{~h}$ 153 vs. in vitro (rich media). Bacterial strains used for co-transcriptomics are shown. 154 (D) Principal component analysis of bacterial gene (orthologous group) 155 expression in planta and in vitro. (E) Gene expression fold changes (in planta vs. 156 in vitro) of bacteria. The data of Pto and D36E are from a previous study (Nobori 157 et al., 2018). Genes not detected or missing in the genome are shown in gray. 158 See Data $\mathbf{S 2}$ for gene expression data. (A, B, D, and E) The taxonomic affiliation 159 (phylum/class level) of each strain is indicated with different colors.

\begin{tabular}{|c|c|c|c|c|c|c|}
\hline ID & Phylum & Class & Order & Family & Genus & RNA-seq \\
\hline Leaf1 & Actinobacteria & Actinobacteria & Actinomycetales & Microbacteriaceae & Microbacterium & P, B \\
\hline Root563 & Actinobacteria & Actinobacteria & Actinomycetales & Intrasporangiaceae & Janibacter & $P$ \\
\hline Soil763 & Actinobacteria & Actinobacteria & Actinomycetales & Micrococcaceae & Arthrobacter & $\mathrm{P}, \mathrm{B}$ \\
\hline Leaf176 & Bacteroidetes & Sphingobacteriia & Sphingobacteriales & Sphingobacteriaceae & Pedobacter & $\mathrm{P}, \mathrm{B}$ \\
\hline Leaf404 & Bacteroidetes & Flavobacteriia & Flavobacteriales & Flavobacteriaceae & Chryseobacterium & $\mathrm{P}, \mathrm{B}$ \\
\hline Root935 & Bacteroidetes & Flavobacteriia & Flavobacteriales & Flavobacteriaceae & Flavobacterium & $\mathrm{P}, \mathrm{B}$ \\
\hline Leaf1 87 & Firmicutes & Bacilli & Bacillales & Exiguobacterium & Exiguobacterium & $\mathrm{P}, \mathrm{B}$ \\
\hline Root147 & Firmicutes & Bacilli & Bacillales & Bacillaceae & & $P$ \\
\hline Leaf155 & Proteobacteria & Alphaproteobacteria & Rhizobiales & Rhizobiaceae & Agrobacterium & $\mathrm{P}, \mathrm{B}$ \\
\hline Leaf177 & Proteobacteria & Betaproteobacteria & Burkholderiales & Burkholderiaceae & Burkholderia & $\mathrm{P}, \mathrm{B}$ \\
\hline Leaf53 & Proteobacteria & Gammaproteobacteria & Enterobacteriales & Enterobacteriaceae & Erwinia & $P$ \\
\hline Leaf70 & Proteobacteria & Gammaproteobacteria & Xanthomonadales & Xanthomonadaceae & Xanthomonas & $P$ \\
\hline Leaf148 & Proteobacteria & Gammaproteobacteria & Xanthomonadales & Xanthomonadaceae & Xanthomonas & $P$ \\
\hline Root604 & Proteobacteria & Gammaproteobacteria & Xanthomonadales & Xanthomonadaceae & & $\mathrm{P}$ \\
\hline Leaf48 & Proteobacteria & Gammaproteobacteria & Pseudomonadales & Pseudomonadaceae & Pseudomonas & $P$ \\
\hline Leaf58 & Proteobacteria & Gammaproteobacteria & Pseudomonadales & Pseudomonadaceae & Pseudomonas & $P$ \\
\hline Leaf127 & Proteobacteria & Gammaproteobacteria & Pseudomonadales & Pseudomonadaceae & Pseudomonas & $P$ \\
\hline Leaf130 & Proteobacteria & Gammaproteobacteria & Pseudomonadales & Moraxellaceae & Acinetobacter & $\mathrm{P}, \mathrm{B}$ \\
\hline Pto & Proteobacteria & Gammaproteobacteria & Pseudomonadales & Pseudomonadaceae & Pseudomonas & $\mathrm{P}, \mathrm{B}$ \\
\hline D36E & Proteobacteria & Gammaproteobacteria & Pseudomonadales & Pseudomonadaceae & Pseudomonas & $\mathrm{P}, \mathrm{B}$ \\
\hline
\end{tabular}

160 Table 1: List of bacterial strains used in this study RNA-seq data ( $P$, plant; $B$, 161 bacteria) obtained in this study are indicated.

\section{Conserved and strain-specific regulation of commensal}

\section{3 functions in planta}

164 The high variability in bacterial genomes complicates a genome-wide comparison 165 of bacterial gene expression among phylogenetically diverse strains (Fig. 1E). To

166 overcome this problem, we sought to compare the regulation of bacterial 167 functions rather than of individual genes. For each strain, we performed 
168 functional enrichment analysis on genes significantly up- or down-regulated in

169 planta using KEGG ontologies (KOs) assigned to individual OGs (see Method for

170 details). Then, enrichment scores ( $p$ values) for individual KOs were summarized

171 for all the strains (Fig. 2A and Fig. S3A). Data from Pto grown in a minimal

172 medium were included to determine the effect of nutrient availability on gene

173 expression changes in a pathogen. A clear pattern distinguishing virulent and

174 avirulent strains was seen in the process "ribosome" (Fig. 2A and 2B). Genes

175 encoding ribosomal subunits were significantly suppressed in planta in all the

176 commensal strains tested and the avirulent pathogen D36E, while these genes

177 were induced in the virulent pathogen Pto (Fig. 2A and 2B). The population

178 density of Pto remains unchanged at this time point (Nobori et al., 2018),

179 indicating that the gene expression changes are not the consequence of

180 bacterial growth in planta. This process was also suppressed in Pto grown in a

181 minimal medium compared with a rich medium (Fig. 2A and 2B). Similarly,

182 genes encoding proton ATPases, which are involved in energy production, were

183 induced in Pto in planta, but suppressed or not altered in the commensal strains

184 (Fig. 2A and 2B). Together, these results suggest that commensal strains are

185 metabolically less active in planta compared with a virulent pathogen, which is

186 consistent with the notion that commensal strains do not proliferate in plants to

187 the same extent as virulent pathogens. Since D36E is a mutant of Pto lacking

188 PTI-suppressing effector molecules, PTI is likely responsible for suppressing

189 bacterial metabolism in planta, at least in this strain. Catalase genes were

190 commonly induced in most commensals and D36E at varying degrees but not in

191 Pto (Fig. 2C), suggesting that commensals are responding to plant ROS burst, a

192 characteristic PTI response. 
A

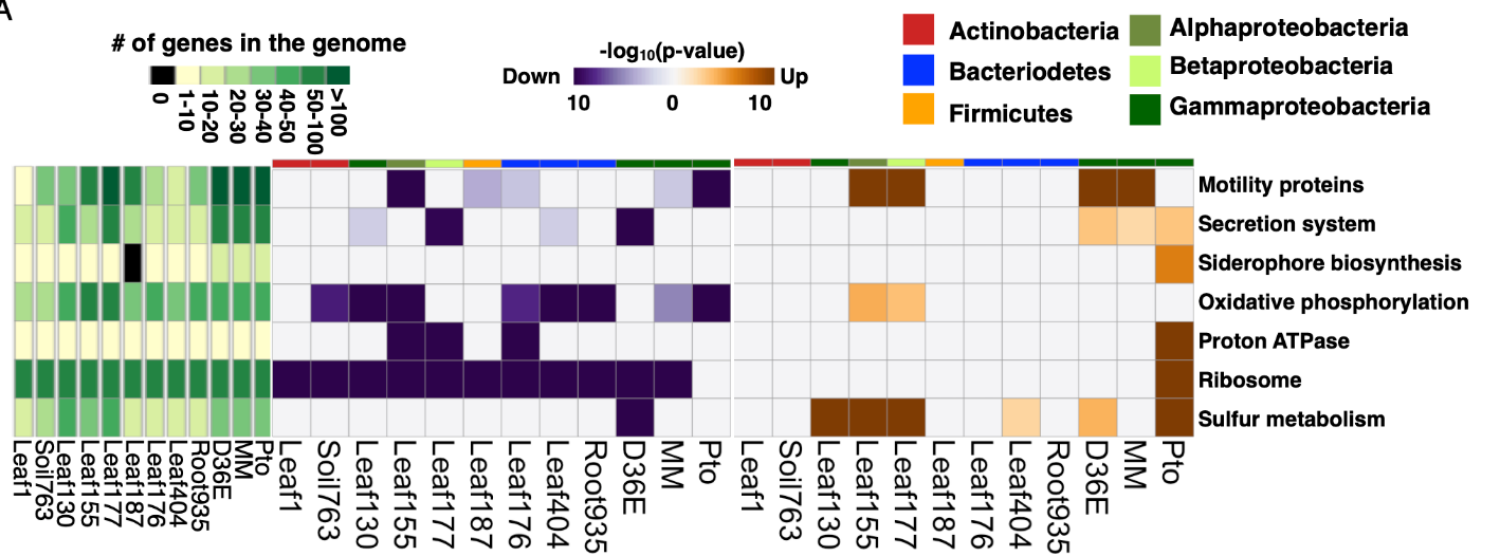

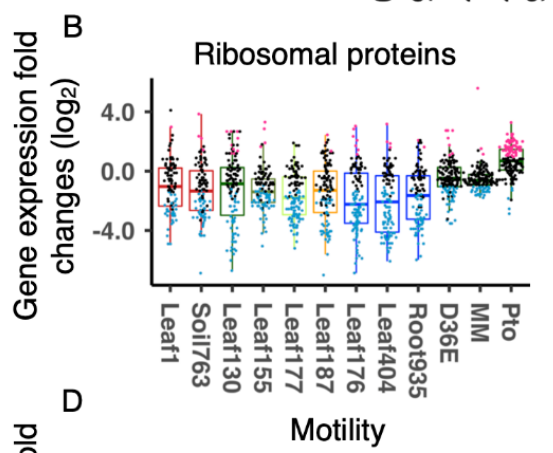

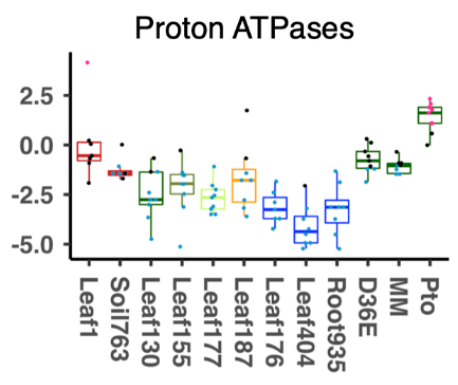

Flagellar assembly proteins
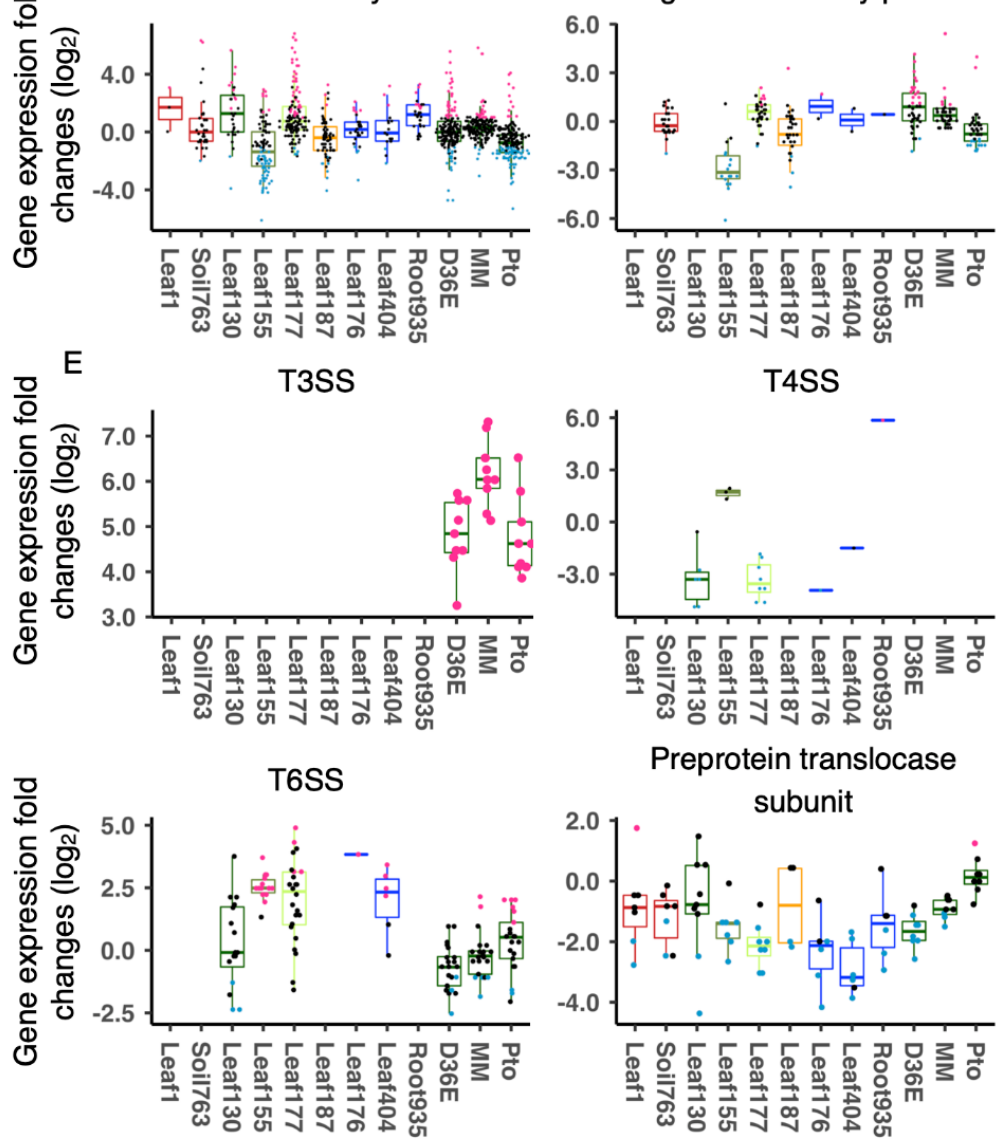

C

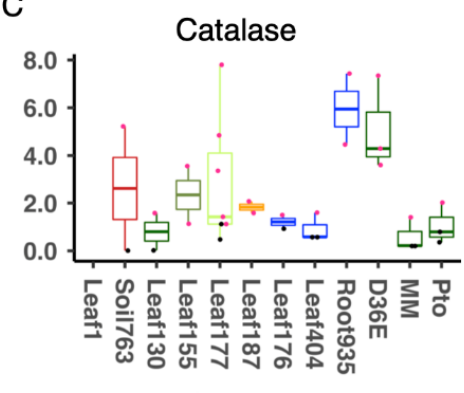

Chemotaxis

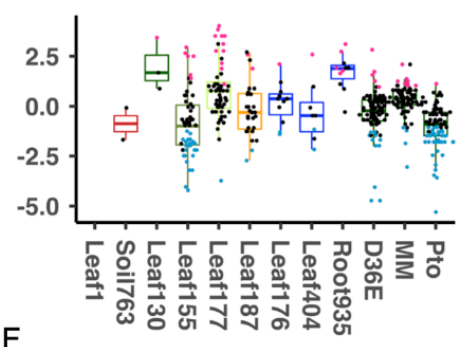

Siderophore biosynthesis

G Function unknown

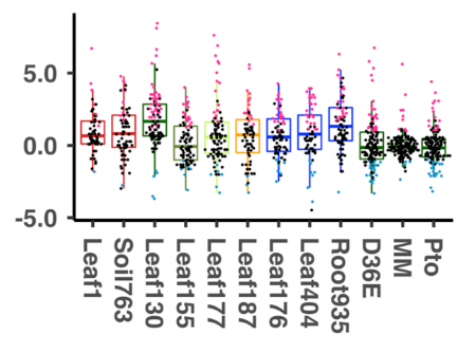


193 Fig. 2: Conserved and strain-specific regulation of bacterial functions in

194 plants (A) KEGG ontology (KO) terms enriched in genes that are significantly

195 up- (orange) or down (purple)-regulated in planta compared with in vitro (rich

196 media). The heatmaps indicate $-\log _{10} p$-value (FDR corrected by

197 Benjamini-Hochberg method). A KO can be both significantly up and

198 down-regulated in the same strain. The left green panel shows the number of

199 genes involved in each KO term. The top color bars indicate the taxonomic

200 affiliation (phylum/class level) of each strain. See Fig. S2 for a more

201 comprehensive list of KOs. (B-G) Expression fold changes (in planta vs. in vitro)

202 of genes involved in (B) general metabolism, (C) catalase metabolic pathway, (D)

203 motility, (E) secretion systems, (F) siderophore biosynthesis, and (G) unknown

204 functions. T3SS, type III secretion system. T4SS, type IV secretion system.

205 T6SS, type VI secretion system. MM, Pto grown in a minimal medium. Results

206 are shown as box plots with boxes displaying the 25th-75th percentiles, the

207 centerline indicating the median, whiskers extending to the minimum, and

208 maximum values no further than 1.5 inter-quartile range. All individual data points

209 (genes) are overlaid with colors for DEGs (red: upregulated, blue:

210 downregulated, black: non-DEG).

211 Genes involved in bacterial motility were differentially regulated among

212 bacteria in plants. Many of these genes were suppressed in Pto in planta but

213 induced in D36E (Fig. 2A and 2D). Leaf177, a Burkholderia (Betaproteobacteria)

214 strain, showed a similar pattern to D36E (Fig. 2A and 2D). However, the

215 Rhizobia (Alphaproteobacteria) Leaf155 more closely resembled virulent Pto - a

216 majority of the genes were suppressed in planta, whereas some genes were

217 significantly induced (Fig. 2D). Motility-related genes can be classed into two

218 major functional categories, flagellar assembly, and chemotaxis. Genes encoding

219 flagellar assembly proteins were globally suppressed in planta in Leaf155 as in

220 Pto, and many Leaf177 chemotaxis-related genes were induced in planta in

221 contrast to Pto (Fig. 2D). Thus, physiological processes are differentially

222 regulated among different plant-associated commensal bacteria strains, with

223 some species even exhibiting similarity to a virulent pathogen.

224 The type III secretion system, an essential component of the virulence of

225 bacterial pathogens, including Pto (Toruño et al., 2016), was strongly induced in

226 Pto and D36E, but these genes were absent in the commensals (Fig. 2E). The 
227 type IV secretion system is involved in multiple processes such as translocating

228 proteins and DNA into other cells and bacterial motility (Costa et al., 2015). This

229 process was globally suppressed in Leaf130 and Leaf177, but not in Leaf155

230 (Fig. 2E). The type VI secretion system is an injection machine involved in

231 bacteria-host and bacteria-bacteria interactions (Russell et al., 2014). This

232 machinery was globally induced in Leaf155 and Leaf404 and partially induced in

233 Leaf177 and Pto (Fig. 2E). Lastly, preprotein translocase subunits, which are

234 involved in the bacterial general secretory pathway (Osborne et al., 2005),

235 tended to be suppressed in all commensals and D36E, but not in Pto (Fig. 2E).

236 It has been shown that genes encoding iron-chelating siderophores are

237 strongly induced in Pto upon plant infection, and the induction of these genes is

238 blocked by plant immunity to suppress bacterial growth (Nobori et al., 2018) (Fig.

239 2F). Commensal strains either harbor only a few or completely lack genes

240 encoding siderophores, and most of these genes were not induced in planta (Fig.

241 2F). We speculate that siderophores are not actively used by commensals in

242 leaves and/or that plant immunity suppresses the production of siderophores to

243 control commensal growth. Notably, many genes annotated as "Function

244 unknown" were significantly induced in planta in various commensals (Fig. 2G).

245 These functionally unannotated genes induced in planta may have unique roles

246 in plant-bacterial interactions.

\section{Phylum and strain-specific gene expression}

248 To compare expression of individual genes between different strains, we 249 conducted comparative transcriptome analysis focusing on specific phyla (Fig. 250 S4A and S4C). This approach allows more comprehensive comparative 251 transcriptome analysis as more genes are shared among closely related strains.

252 We focused on Bacteroidetes and Proteobacteria, in which 1,422 and 1,122 OGs 253 were shared, respectively (far more than the 454 OGs shared among the nine 
254 commensals) (Fig. S2B, S4A, and S4C). Overall, many genes were differentially

255 regulated in a single strain (Fig. S2B, S4A, and S4C). In both of these phyla, a

256 larger number of genes were commonly suppressed among the three strains in

257 plants than commonly induced (Fig. S4B, S4D, and S5). Clusters of genes

258 commonly suppressed in planta (clusters 7 \& 8 in Bacteroidetes and clusters 1 \&

2594 in Proteobacteria) were enriched with "ribosome"-related genes (Fig. S4E).

260 "Transporters" were enriched in multiple clusters with various expression patterns

261 (Fig. S4F and S6A), suggesting that transporters can be separated into

262 sub-groups based on regulation in plants. Also, genes annotated as part of a

263 "two-component system" showed strain-specific expression patterns (Fig. S4G

264 and S6B). Taken together, our intraphylum analysis reveals that even relatively 265 closely related commensal strains respond differently in planta at the 266 transcriptional level.

267 In planta bacterial transcriptomics illuminates bacterial 268 adaptation to the leaf environment

269 Various bacterial functions were differentially regulated in plants in a 270 strain-specific manner. Is such functional regulation relevant for bacterial fitness

271 in plants? Comparative genomics is one way to infer bacterial functions

272 associated with adaptation to the plant environment. A previous study compared

273 the genomes of nearly 4,000 plant-associated and non-plant-associated bacterial

274 strains and defined "plant-associated (PA) genes" that are significantly enriched

275 in plant-associated strains (Levy et al., 2017). We analyzed how PA genes are

276 regulated in plants in our transcriptome data. When analyzing the genes shared

277 among nine commensal strains, we observed that genes induced in planta

278 tended to be enriched with PA genes, whereas genes suppressed in planta

279 tended to be enriched with nonPA genes (Fig. 3A). Remarkably, PA and nonPA

280 genes were significantly enriched with plant-induced and plant-suppressed 
281 genes, respectively, for all the commensals, except for the Firmicutes strain 282 Leaf187 (Fig. 3B). Therefore, our data suggest that bacterial genes associated 283 with adaptation to the plant environment were indeed activated in planta.

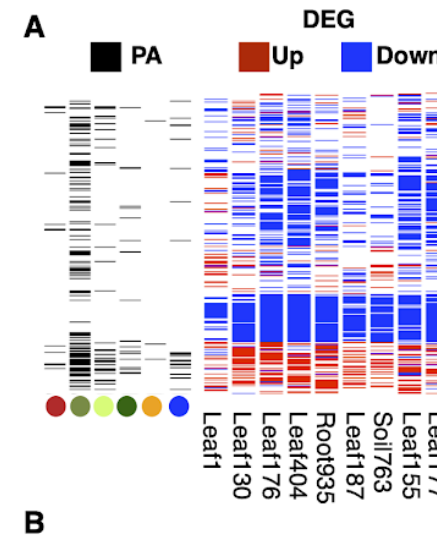

Leaf1
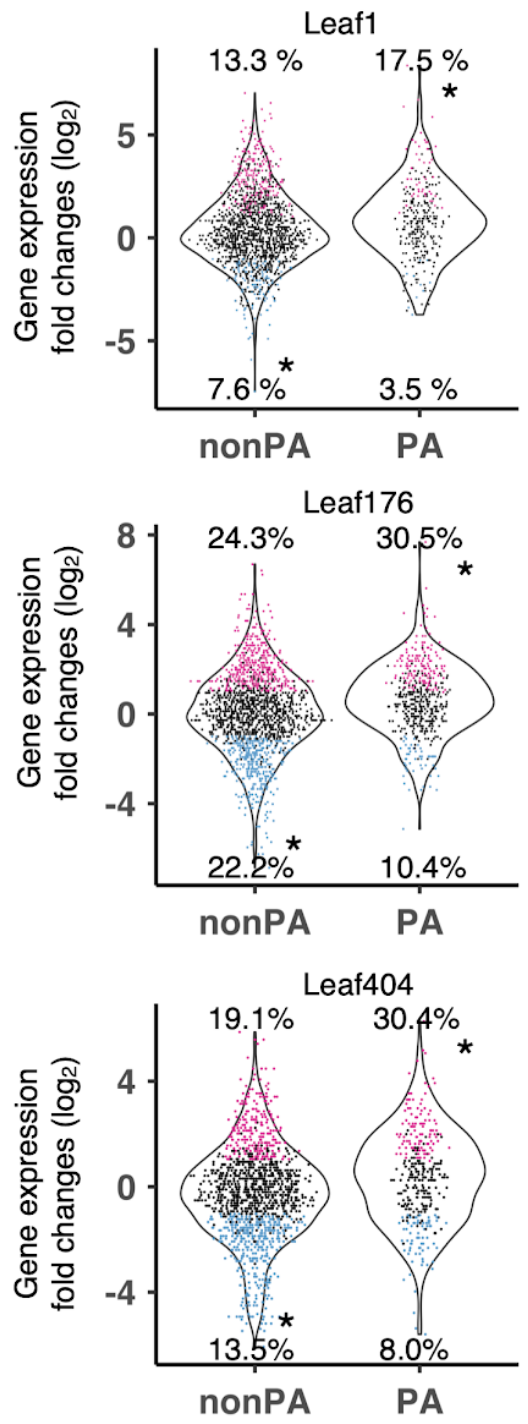
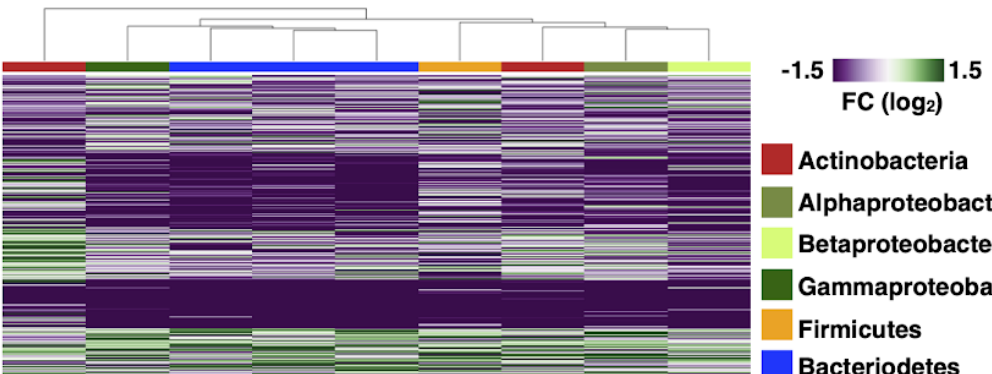

Actinobacteria

Alphaproteobacteria Betaproteobacteria Gammaproteobacteria Firmicutes Bacteriodetes

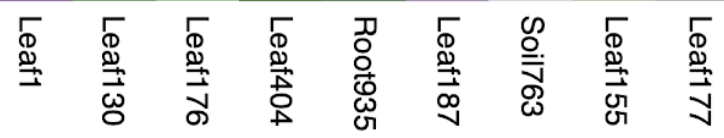
Leaf130
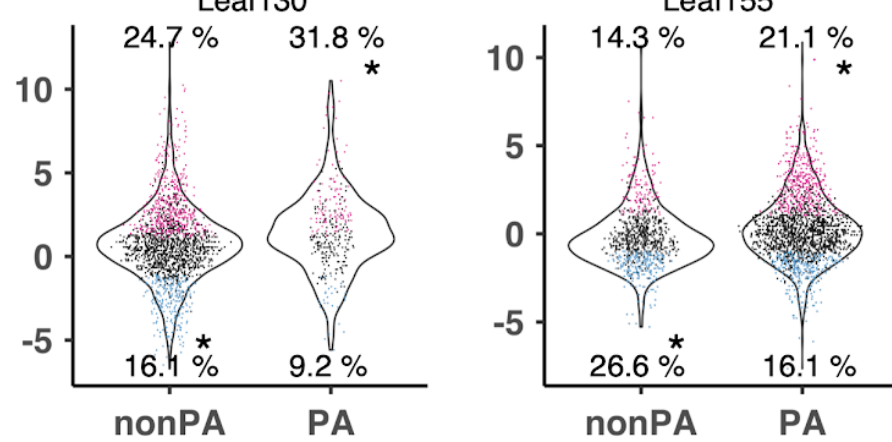

Leaf177
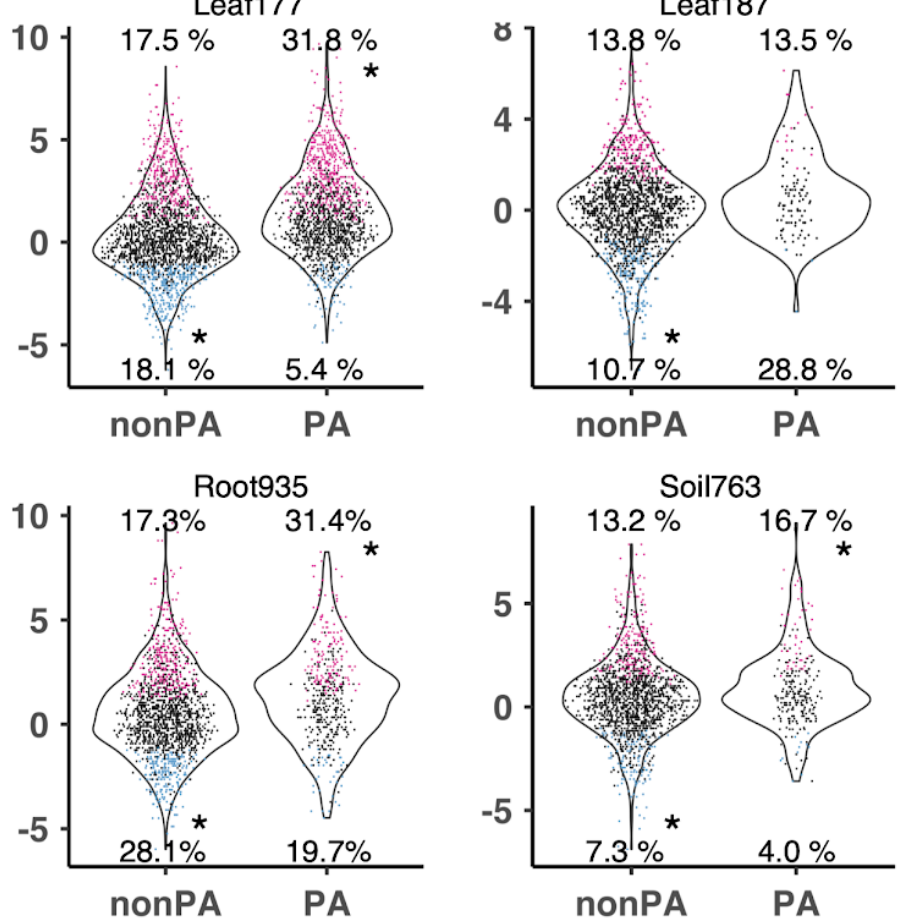

Nobori et al. | Co-transcriptomics of plants and microbiota 
284 Fig. 3: Genes enriched in plant-associated bacteria are induced in planta 285 (A) (Right panel) Bacterial gene expression fold changes (FC) in plants 286 compared with in vitro (rich media). (Middle panel) Genes differentially expressed 287 in planta compared with in vitro $\left(\left|\log _{2} \mathrm{FC}\right|>1\right.$; FDR $<0.01$; two-tailed Student's $\mathrm{t}$ 288 test followed by Storey's q-value). (Left panel) Genes previously shown to be 289 "plant-associated" (Levy et al., 2017) are shown as black. The bar and dots 290 indicate the taxonomic affiliation (phylum/class level) of each strain. (B) Boxplots 291 showing expression changes of plant-associated (PA) and non-plant-associated 292 (nonPA) genes between in planta and in vitro. Each dot represents a gene. 293 Genes significantly up- or downregulated in planta are colored in red and blue, 294 respectively. Asterisks indicate statistically significant enrichment (FDR < 0.05; 295 Hypergeometric test corrected by Benjamini-Hochberg method) of up or 296 down-regulated genes in the PA or nonPA category. The proportion of genes up297 or downregulated are shown. For the full expression data with the orthologous 298 group, KEGG annotation, DEG, and PA information, see Data S3.

299 We then performed KO enrichment analysis for PA genes induced in plants 300 and nonPA genes suppressed in plants. Ribosome-related genes were 301 conserved among all strains (and are thus nonPA genes) and were generally 302 suppressed in plants (Fig. 4A), which may be a strategy by which plants control 303 bacterial growth. Glycan degradation genes were highly plant-associated and 304 induced in Bacteroidetes strains Leaf176 and Root935 (Fig. 4A and 4B). Among 305 such genes were homologs of beta-galactosidase, alpha-L-fucosidase, and 306 glucosylceramidase, which can degrade plant cell wall components. Thus,

307 Leaf176 and Root935 may have evolved the ability to degrade the plant cell wall 308 enabling the establishment of favorable niches during plant colonization.

309 "Sulfur metabolism"-related genes were classified as PA genes and were 310 induced in planta in the three Proteobacteria strains (Fig. 4A and 4C). "ABC 311 transporters" was another PA function that was induced in Proteobacteria strains 312 in planta (Fig. 4A and 4C). Genes involved in both categories were sulfur uptake 313 transporters (Fig. 4C). A previous proteomics study showed that the expression 314 of proteins involved in sulfur metabolism and uptake was induced on the leaf 315 surface in two commensal Proteobacteria, Sphingomonas melonis and 
316 Methylobacterium extorquens (Müller et al., 2016). These results suggest that

317 sulfate acquisition is important for the adaptation of commensal Proteobacteria to

318 the plant environment. On the other hand, sulfur metabolism-related genes were

319 not found to be PA genes and were suppressed in planta in the Bacteroidetes

320 strains Leaf176 and Leaf404 (Fig. 4A and 4C). The number of genes predicted

321 to be involved in sulfur metabolism was lower in Bacteroidetes strains than in

322 Proteobacteria strains (Fig. 4A). This may indicate that Bacteroidetes strains are

323 less reliant on sulfur acquisition during plant colonization.

324 Urea transporters are other ABC transporters that are PA genes and were 325 induced specifically in some Proteobacteria strains in planta (Fig. 4D). Also,

326 genes encoding ureases, which hydrolyze urea in the bacterial cytoplasm, were 327 induced in some Proteobacteria strains (Fig. 4D). It has been shown that

328 Yersinia enterocolitica, a Gammaproteobacteria strain, can use urea as a 329 nitrogen source (Young et al., 1996). These results suggest that Proteobacteria 330 (especially Leaf177 and D36E) might require urea as a nitrogen source in the 331 plant apoplast. Also, genes involved in the nitrate transport system were induced 332 in Leaf130, Leaf155, and D36E in planta, but not in Pto (Fig. S7), suggesting that 333 some commensal Proteobacteria strains activate nitrogen acquisition systems in 334 plants. Similarly, ribose transporters and glycerol transporters are PA genes and 335 were commonly induced in planta in commensal or avirulent Proteobacteria 336 strains (Leaf155, Leaf177, and D36E) but not in the virulent Pto (Fig. 4F). 337 Moreover, arabinose and xylose transporters (both monosaccharide transporters) 338 were induced in Leaf177 and D36E in planta, but not in Pto (Fig. S7). Thus, 339 these Proteobacteria strains may use various types of sugars as carbon sources 340 in plants. The induction of urea and sugar acquisition systems may indicate that 341 commensal bacteria activate nutrient starvation responses in the leaf apoplast.

342 We speculate that plants may sequester nitrogen and carbon sources from the 343 apoplast to limit the growth of commensal and avirulent pathogenic bacteria. In 344 line with this hypothesis, the high-affinity plant urea transporter AtDUR3 was 
345 induced upon inoculation with many commensal strains while suppressed by the

346 virulent Pto (Fig. 4E). A previous study showed that plants sequester

347 extracellular sugars by activating the sugar influx transporter AtSTP13 via the

348 PTI pathway (Yamada et al., 2016). Indeed, our plant transcriptome data showed

349 that AtSTP13 is induced by the commensals as well as D36E and Pto (Fig. 4G).

350 On the other hand, Pto can induce plant sugar efflux transporters (Chen et al.,

351 2010), which might increase sugar availability in the apoplast and explain why

352 Pto did not activate its sugar transporters in plants. A non-mutually exclusive

353 possibility is that the virulent Pto switches its metabolic preference to other

354 substrates during successful infection in plants. In summary, we revealed

355 bacterial phylum/strain-specific gene repertoires and gene regulation, which may

356 be actively controlled by plants and drive bacterial niche separation in planta. 
A

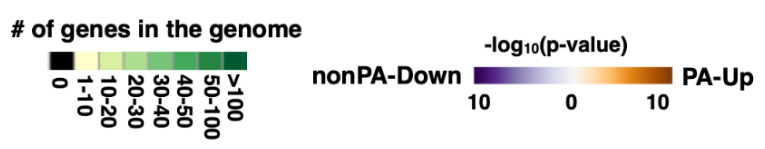

357
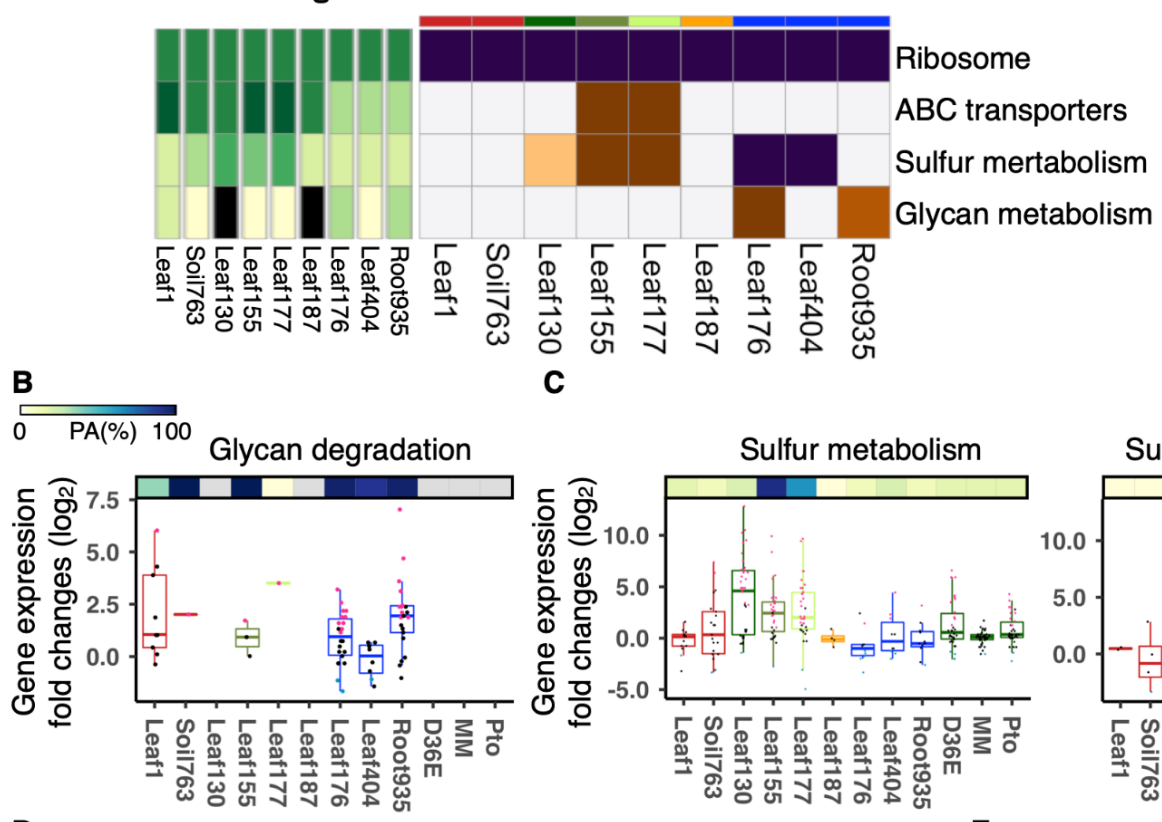

Actinobacteria

Bacteriodetes

Firmicutes

Alphaproteobacteria

Betaproteobacteria

Gammaproteobacteria

B

D

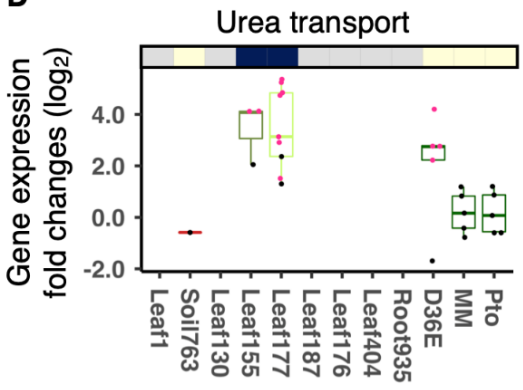

F

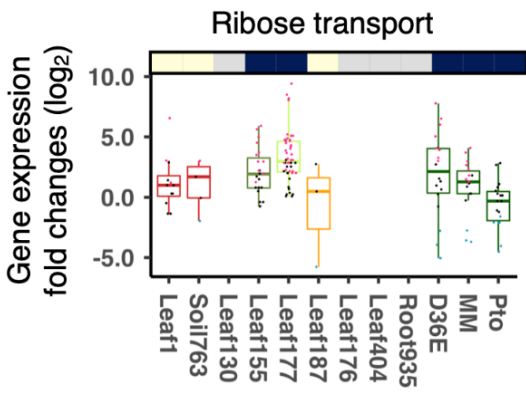

C

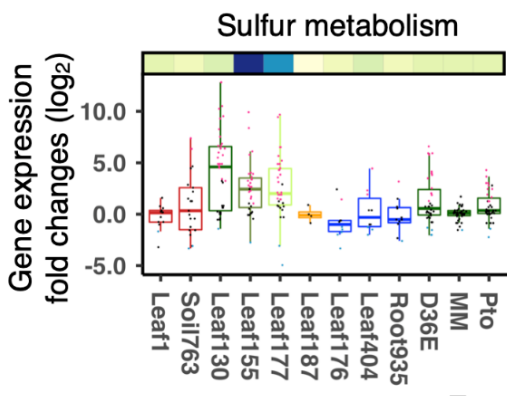

Sulfur metabolism

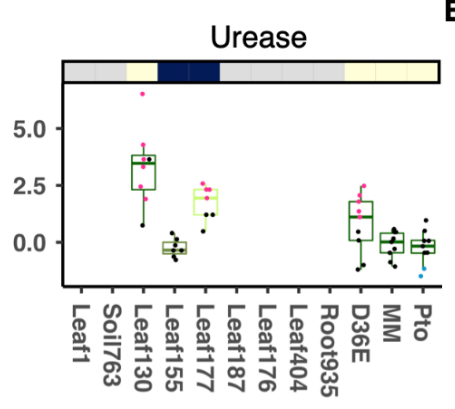

E

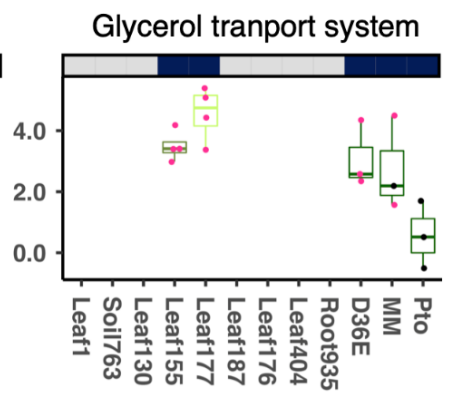

Sulfur uptake transporters
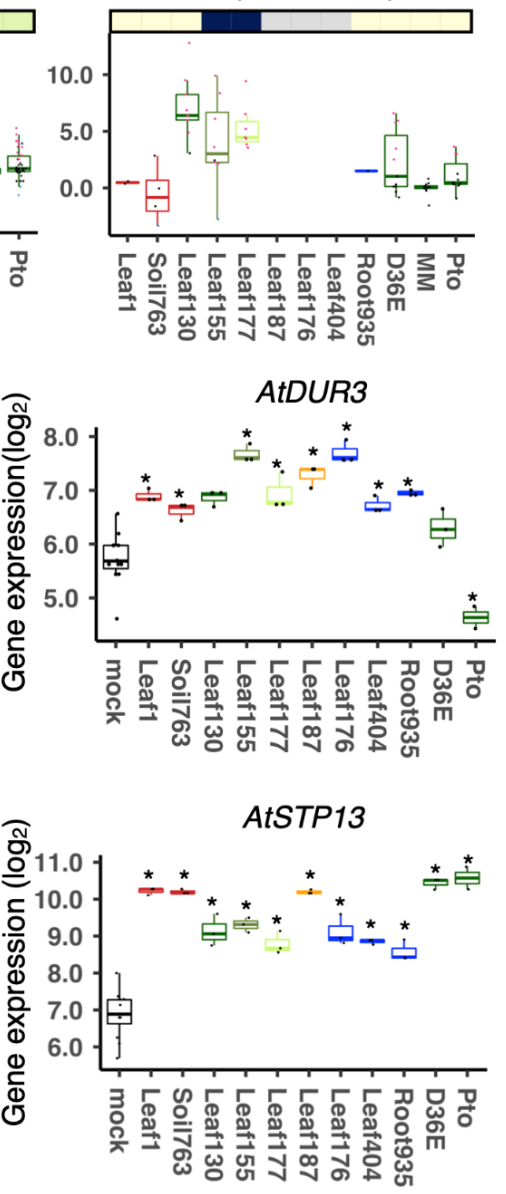

358 Fig. 4: Nutrient acquisition systems are associated with bacterial 359 adaptation to the plant environment in a strain-specific manner (A) KEGG 360 enrichment analysis of genes that are plant-associated (PA) and significantly 361 induced in planta compared with in vitro (rich media) (orange) and genes that are 362 nonPA and significantly suppressed in planta compared with in vitro (purple). The 363 left panel shows the number of genes involved in each KEGG term. (B-D, F) 364 Expression fold changes (in planta vs. in vitro) of genes with different functions. 
365 The top bars indicate the ratio of PA genes in each strain. All individual data 366 points (genes) are overlaid to the box plots with colors for DEGs (red: 367 upregulated, blue: downregulated, black: non-DEG). (E and G) Expression of the 368 plant gene (E) AtDUR3 (urea transporter) and (G) AtSTP13 (sugar transporter) 369 based on the RNA-seq data. Asterisks indicate statistically significant difference $370\left(\left|\log _{2} \mathrm{FC}\right|>1\right.$; FDR $<0.01$; two-tailed Student's $\mathrm{t}$ test followed by Storey's 371 q-value) compared with the mock (water-inoculated) condition. In the box plots, 372 boxes display the 25th-75th percentiles, the centerline indicating the median, 373 whiskers extending to the minimum, and maximum values no further than 1.5 374 inter-quartile range. For the full expression data with the orthologous group,

375 KEGG annotation, DEG, and PA information, see Data S3.

376 Commensals activate plant PTI in a strain-specific manner

377 As commensal strains showed differing responses in plants, indicating strain 378 specificity in the interactions of plants with bacteria, we investigated 379 genome-wide plant responses to individual commensals by RNA-seq. In addition 380 to the nine strains used for the bacterial transcriptome analysis, we added nine 381 more commensal strains to enrich our dataset. Global gene expression changes 382 (bacteria-inoculated vs. water-inoculated) were similar among all commensal 383 strains as well as D36E at 6 hpi (Fig. 5A). Interestingly, plant gene expression 384 changes triggered by commensals overlapped markedly with responses to flg22 385 (Hillmer et al., 2017), a potent PTI inducer. PTI-inducible genes accounted for 386 clusters of genes commonly and strongly induced by most of the commensals 387 (clusters 3 \& 5 in Fig. 5A). GO enrichment analysis showed that these clusters 388 are enriched with genes related to defense responses (Fig. 5B). Thus, 389 commensal strains, when infiltrated into plant leaves, can induce MTI.

390 The degree of PTI induction varied among strains in a manner that is partly 391 determined by phylogeny: Gammaproteobacteria and Actinobacteria strains 392 induced stronger PTI than Bacteroidetes strains (Fig. 5C). We then investigated 393 the amino acid sequences of the major MAMPs flg22 and elf18 across the 394 different strains. Intriguingly, strains with flg22 and elf18 sequences similar to 395 those known to be particularly potent PTI inducers (Felix et al., 1999; Kunze et 396 al., 2004) tended to elicit strong PTI induction (gene expression fold changes in 
bioRxiv preprint doi: https://doi.org/10.1101/2021.04.25.440543; this version posted April 26, 2021. The copyright holder for this preprint (which was not certified by peer review) is the author/funder, who has granted bioRxiv a license to display the preprint in perpetuity. It is made available under aCC-BY-NC-ND 4.0 International license.

397 clusters 3 or 5) (Fig. 5D). Thus, sequence variation in these MAMPs may partly

398 determine the degree of PTI induced by some of these commensal strains.

A

\begin{tabular}{|l|l|l}
\hline Actinobacteria & Firmicutes & Betaproteobacteria \\
\hline Bacteriodetes & Alphaproteobacteria & Gammaproteobacteria
\end{tabular}

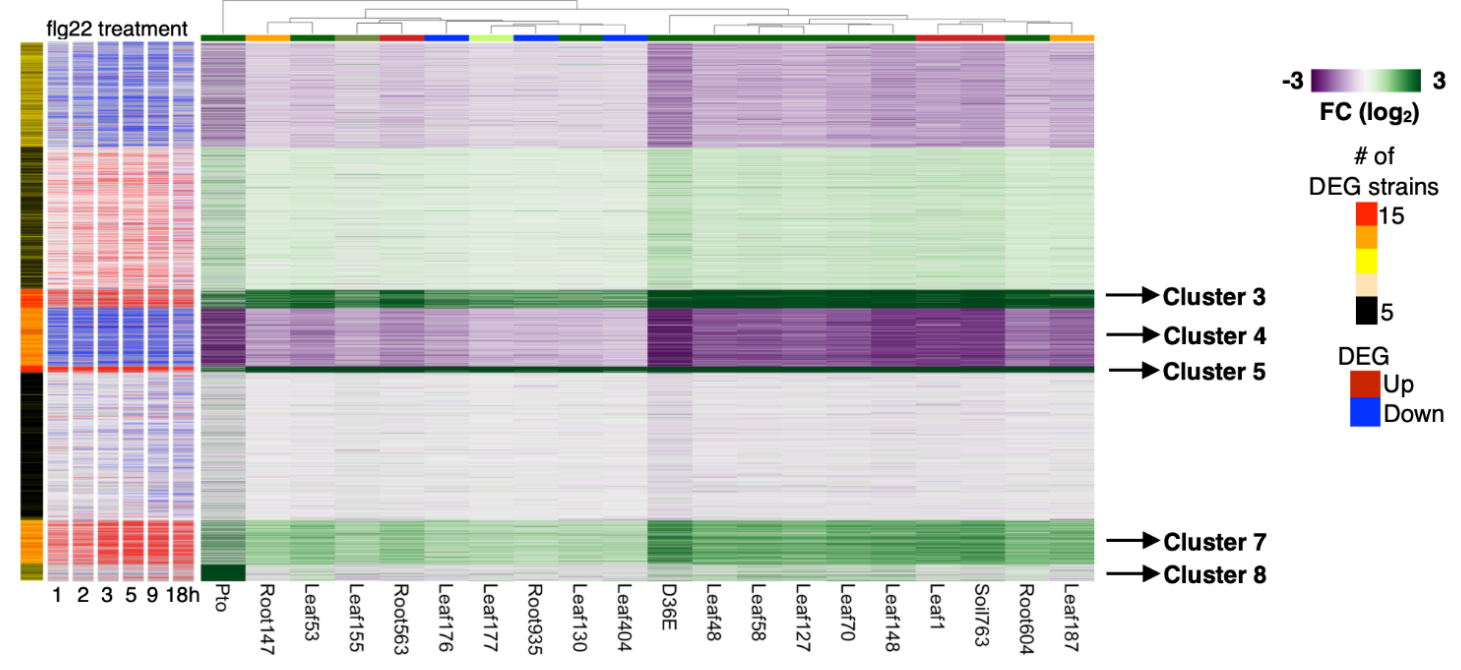

B

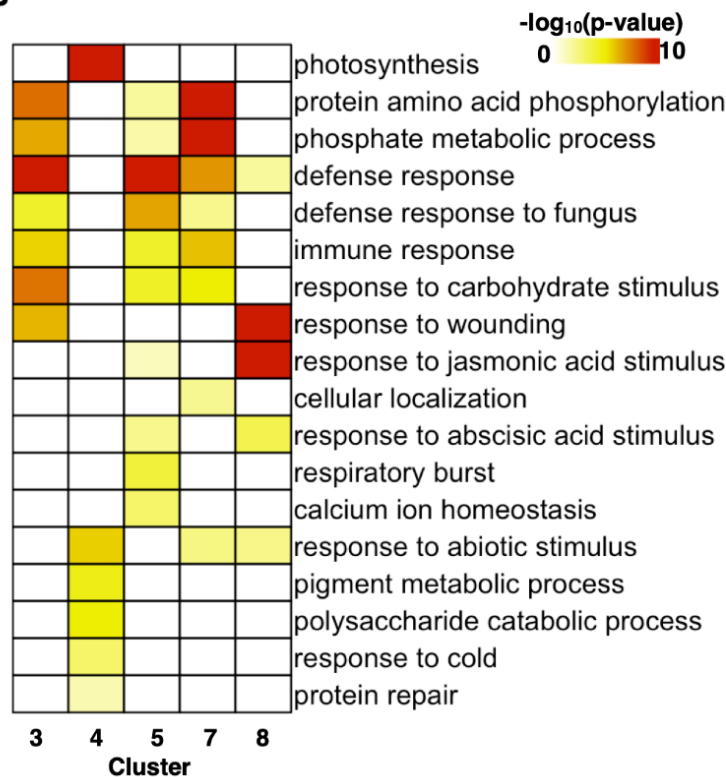

C

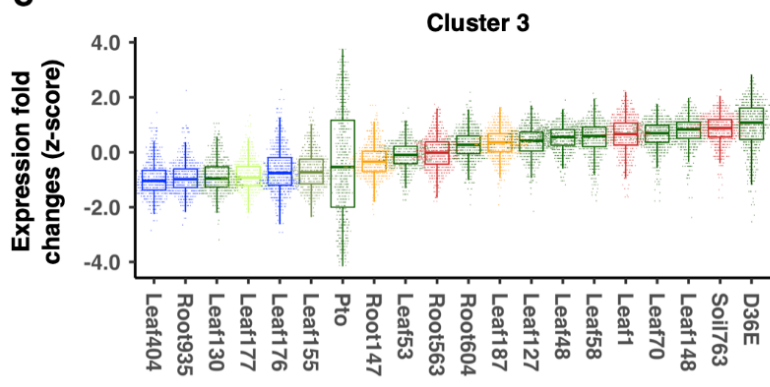

D
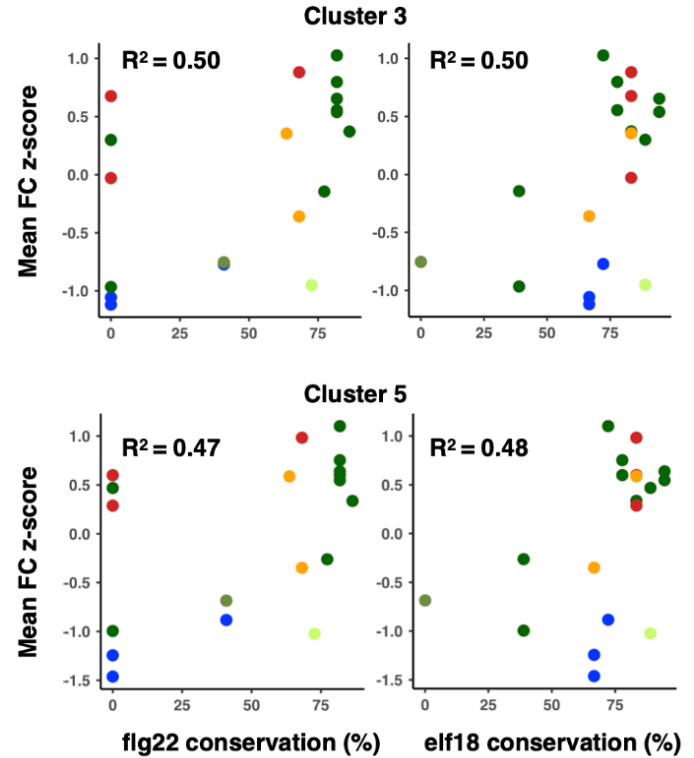

Cluster 5

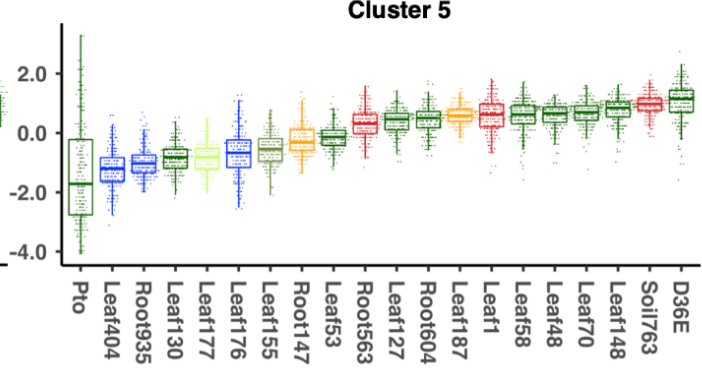


399 Fig. 5: Plant transcriptome responses to phylogenetically diverse 400 commensals (A) (Green/purple heatmap) Gene expression fold changes (FCs) 401 between bacteria-inoculated plants and water-inoculated plants. (Red/blue 402 heatmap) Plant genes significantly induced or suppressed upon flg22 treatment 403 at different time points (Hillmer et al., 2017). The number of strains causing 404 differential gene expression $\left(\left|\log _{2} \mathrm{FC}\right|>1\right.$; FDR $<0.01$; two-tailed Student's t test 405 followed by Storey's q-value) are indicated in the sidebar (\# of DEG strains). 406 DEG, differentially expressed gene. Genes were clustered by k-mean clustering $407(\mathrm{k}=8)$. The bars on the heatmaps indicate the taxonomic affiliation (phylum/class 408 level) of each strain. See Data S4 for gene expression data. (B) Gene ontology 409 enrichment analysis for genes in clusters 3, 4, 5, 7, and 8 of (A). $-\log _{10}$ p-values 410 (FDR corrected by Benjamini-Hochberg method) were shown. (C) Expression 411 fold changes (FC; z-score) of genes in clusters 3 and 5 . Results are shown as 412 box plots with boxes displaying the 25th-75th percentiles, the centerline 413 indicating the median, whiskers extending to the minimum, and maximum values 414 no further than 1.5 inter-quartile range. (D) Relationships between amino acid 415 (AA) sequence conservation of flg22 or elf18 and normalized expression FCs of 416 genes in clusters 3 and 5. AA sequence conservation of flg22 and elf18 417 compared with the canonical sequences known to induce strong defense 418 responses in plants (Elf18: SKEKFERTKPHVNVGTIG. Flg22: 419 QRLSTGSRINSAKDDAAGLQIA). The Pearson correlation coefficients are 420 shown. (C-D) The same color code was used for the taxonomic affiliation.

421 Plant responses do not correlate with bacterial responses in plants

422 Principal component analysis showed that plant and bacterial transcriptome 423 patterns were incongruent with each other (Fig. 6A). For instance, we observed 424 similarity between the plant transcriptome changes elicited by different 425 Actinobacteria strains (Leaf1 and Soil763), but the strains themselves respond 426 highly differently in planta (Fig. 6A). Also, Bacteroidetes strains (Leaf176, 427 Leaf404, and Root935) showed similar transcriptional changes in plants, but 428 plant transcriptome changes triggered by these strains differed more (Fig. 6A). 429 To get deeper insights into the relationships between plant and bacterial gene 430 expression, we measured the correlation between gene expression changes of 431 individual plant genes and shared bacterial OGs using co-transcriptome data of 432 nine commensal strains. To prevent a single outlier strain from impacting 433 correlation scores, we took a bootstrapping approach in which correlations were 434 calculated using all the combinations of eight strains as well as all the nine 
435 strains and then combined (see Methods in detail) (Fig. 6B and Fig. S8). This

436 analysis revealed that the expression of a majority of plant and bacterial genes is

437 not correlated, further indicating that the plant and bacterial responses are largely

438 uncoupled in our dataset (Fig. 6C). For instance, in many cases, commensal

439 strains that triggered similar plant transcriptional responses (e.g., Soil763 and

440 Leaf1; Fig. 6C) showed distinct gene expression in plants (Fig. 6C). However, a

441 subset of plant and bacterial genes showed a stronger correlation (Fig. 6C). KO

442 enrichment analysis showed that expression of genes annotated as belonging to

443 the bacterial processes "proton ATPases" and "purine metabolism" positively

444 correlates with plant defense-related genes (Fig. 6C). More specifically, the

445 expression of such bacterial genes was higher when plants showed stronger PTI

446 activation. The biological relevance of this observation remains to be tested.

447 Together, our data indicate that plant and bacterial gene expression can be

448 largely uncoupled at an early stage of interaction. 


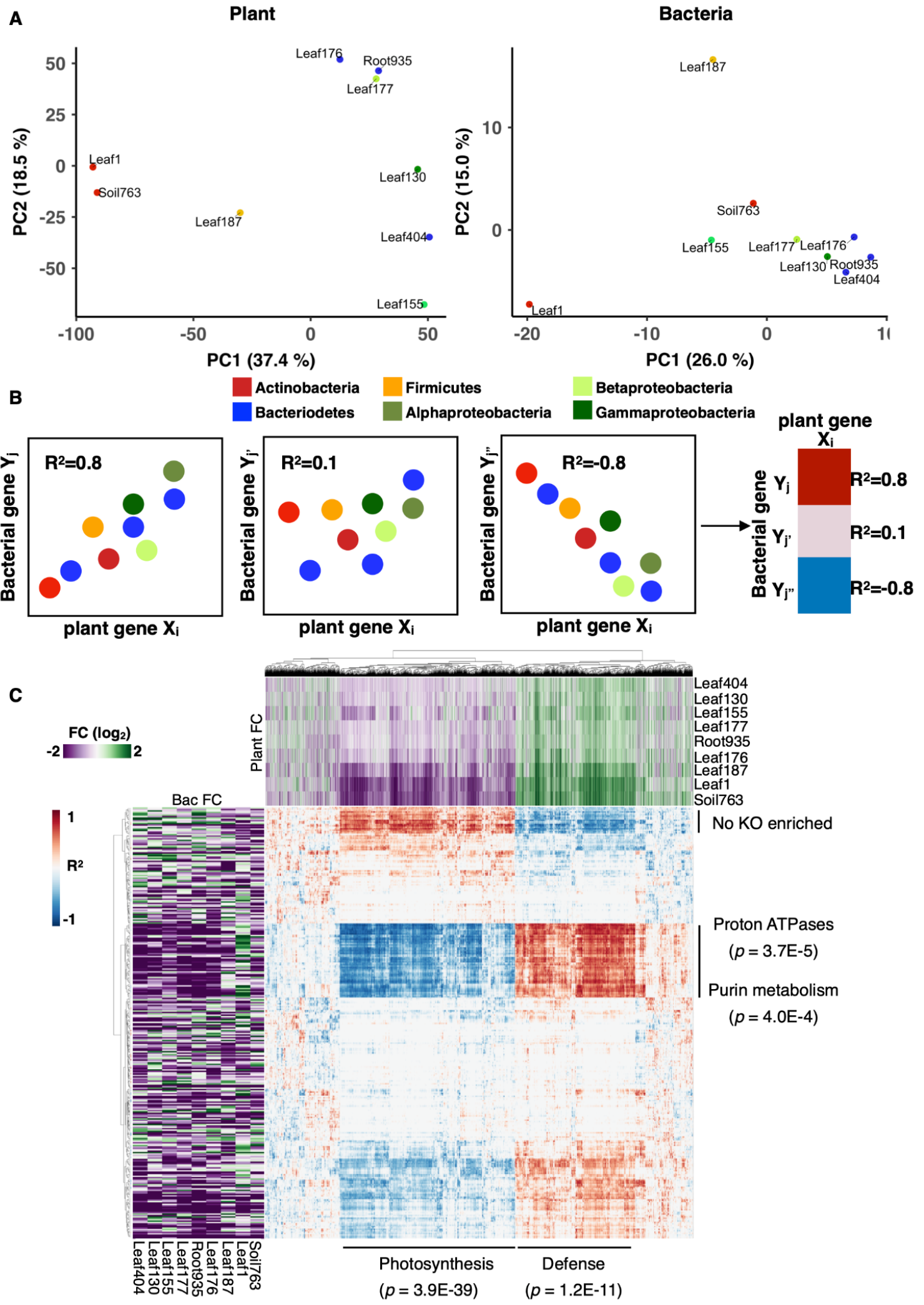


449 Fig. 6: Plant and bacterial transcriptomes are largely uncoupled (A) 450 Principal component analysis of gene expression fold changes (FCs) of plants 451 (left: bacteria-inoculated vs. water-inoculated) and bacteria (right: in planta vs. in 452 vitro). Orthologous groups (OGs) of bacterial genes shared among all strains are 453 used for the analysis. (B) Schematic diagram of the integration of plant and 454 bacterial RNA-seq data. For each interaction condition, the correlation 455 coefficients between individual plant genes and bacterial OGs were calculated. 456 The correlation coefficient data was corrected by bootstrapping (see Methods 457 and Fig. S8) (C) A map of correlation coefficients between plant genes and 458 bacterial OGs calculated as described in (B). Rows and columns are bacterial 459 OGs and plant genes, respectively. The top and left heatmaps indicate gene 460 expression FCs of plants and bacteria, respectively. See Data S5 for the full 461 correlation data. KEGG enrichment analysis was performed for the clusters of 462 plant and bacterial genes with strong correlation.

\section{Discussion}

464 Previous studies of the plant microbiota have suggested that plants assemble 465 bacterial communities and regulate their functions by interacting with 466 commensals in a strain-specific manner. However, there are a limited number of 467 studies that have interrogated the responses of plants and commensal bacteria 468 at a genome-wide scale, and we thus have no comprehensive understanding of 469 the two-way molecular dialogue between plants and microbiota members. Here, 470 we profiled, for the first time, co-transcriptomes of plants and commensal 471 bacteria under monoassociation conditions using diverse strains covering all 472 major phyla of the plant microbiota. Our dataset demonstrated that different 473 commensal strains 1) trigger qualitatively similar yet quantitatively different 474 immune responses in plants and 2) show both common and highly strain-specific 475 responses in plants.

476 We found that suppression of genes related to general metabolic activity and 477 energy production in planta is a common trait among phylogenetically diverse 478 commensals, in marked contrast to a virulent pathogen, which elicited the 479 opposite response (Fig. 2). PTI was commonly induced by the commensal 480 strains (Fig. 5A), suggesting that plant immunity might act to keep commensal 
481 metabolic activity in check to avoid overgrowth. This notion is in line with a

482 previous finding that commensals can proliferate in an unrestrained manner in

483 the leaf apoplast of plant mutants lacking key immune components under high

484 humidity, which further compromises plant immunity (Chen et al., 2020; Xin et al.,

485 2016). Further transcriptome analysis of commensals in immunocompromised

486 plants and environmental conditions will unravel how different immune pathways

487 tailor their responses to effectively control commensal growth and function.

488 We provide evidence that bacterial genes enriched in the genomes of 489 plant-adapted strains are induced in planta (Fig. 3A and 3B), suggesting that 490 those genes which enable bacteria to thrive in the plant environment are indeed 491 activated in plants. This finding is somewhat in contrast to a previous study, 492 which showed that gene expression of a bacterial pathogen in planta does not 493 correlate with fitness scores determined by transposon insertion mutagenesis 494 (Helmann et al., 2019). Importantly, loss-of-function screening with single 495 mutants has limitations in assigning gene function owing to functional 496 redundancy. In this case, a gain-of-function assay is a complementary, albeit also 497 limited, approach. For instance, we previously showed that in planta bacterial 498 transcriptome data can predict bacterial genes that contribute to bacterial growth 499 in planta when overexpressed (Nobori et al., 2018, 2019). It will be necessary, in 500 the future, to rigorously validate the biological relevance of bacterial 501 transcriptional regulation in plants at the mechanistic level.

502 We found that processes involved in the uptake of nutrients, such as sulfur, 503 urea, and sugars, were enriched in plant-associated Alpha- and 504 Betaproteobacteria (L155 and L177) and induced in planta (Fig. 4). We observed 505 that plants induce transporters that could sequester urea and sugars from the 506 apoplast during interactions with commensals (Fig. 4E and 4G), which potentially 507 affects bacterial nutrient acquisition processes and eventually bacterial fitness in 508 plants. Notably, we also found many other bacterial nutrient transporters to be 509 regulated in planta in a strain-specific manner (Fig. S7). The results imply that 
510 different commensals experience distinct nutrient status in the plant apoplast,

511 which might affect bacterial fitness in planta. Our co-transcriptome data sets the

512 stage for investigating whether plants control nutrient availability for particular

513 strains to drive bacterial niche separation in plants and shape the plant

514 microbiota.

515 We did not observe a strong association between gene expression changes in

516 plants and commensals (Fig. 6A and 6C), implying that different commensals

517 could respond divergently to similar programs of plant gene expression. This

518 seems plausible, given that plants have to deal with complex bacterial

519 communities residing in an area smaller than the plant cell. However, our single

520 time point experiment does not allow us to draw a firm conclusion regarding this

521 idea, as there may be a time lag between plant responses and bacterial

522 responses. In the future, time-course analysis of a larger number of strains

523 combined with plant and bacterial genetics will facilitate the prediction of

524 biologically meaningful links between plant and bacterial responses.

525 In this study, bacterial cells were syringe-infiltrated into leaves to bypass

526 stomatal entry as different commensals might have different abilities to access

527 the apoplast. Transcriptomes were profiled at $6 \mathrm{hpi}$, where the population density

528 of even the virulent pathogen Pto has not yet increased (Nobori et al., 2018), and

529 thus we assumed that the population density of commensals remained the same

530 at this time point. Therefore, our experimental setup allowed us to characterize

531 strain-specific co-transcriptomes under controlled conditions without the influence

532 of stomatal immunity and differences in the sizes of bacterial populations. It is,

533 however, important to note that transcriptome analysis under more natural

534 conditions would reveal additional layers of plant-microbiota interactions. This

535 requires technological innovations that enable in planta transcriptome analysis of

536 bacteria with much smaller populations.

537 Since plants used in this study were not grown in a strictly sterile condition,

538 we do not exclude the possibility that the pre-existing plant microbiota influenced 
539 on plant and bacterial responses. However, influence of the pre-existing

540 microbiota on data interpretation was minimized by including the mock control

541 (for plant transcriptomics) and randomizing sampling and taking three

542 independent replicates (for plant and bacterial transcriptomics). We

543 demonstrated that an in planta bacterial transcriptome approach can be applied

544 to all major phyla of the plant microbiota, opening a new avenue for in planta

545 transcriptome analysis of synthetic communities that are generated by mixing

546 bacterial strains in a desired manner. This in planta bacterial metatranscriptome

547 approach can capture more complex traits such as microbe-microbe interactions,

548 which are important to understand the functions of the microbiota as a

549 community.

550 This study has provided a wealth of information regarding gene regulation of

551 both plants and commensals during monoassociations. In Fig. S9-12, we

552 provided additional insights on the regulation of genes related to diverse

553 functions, including biosynthesis/metabolism of various compounds, transporters,

554 and nucleic acid regulation. Notably, we found many genes with unknown

555 functions to be dynamically regulated in commensals during interactions with

556 plants (Fig. 2G). To explore these commensal functions, it will be critical in the

557 future to link bacterial transcriptome responses to bacterial niche preference and

558 reproductive fitness in plants. Our co-transcriptome dataset will provide a robust

559 platform for hypothesis-driven functional investigation of plant and bacterial

560 genes that play critical roles in plant-microbiota interactions. 


\section{Acknowledgments:}

562 We thank Paul Schulze-Lefert and Julia Vorholt for providing the commensal 563 strains, Alan Collmer for providing the Pto DC3000 D36E strain, the Max Planck 564 Genome Centre for sequencing support, Dieter Becker, Ryosuke Kanaoka, 565 Shinpei Shimokawa, and Ying Tang for research assistance, and Neysan 566 Donnelly, You Lu, and Natsuki Ohmae for critical comments on the manuscript. 567 This work was supported by the Fundamental Research Funds for the Central 568 Universities (Program No. 2662020ZKPY009) (to K.T.), the Huazhong 569 Agricultural University Scientific \& Technological Self-innovation Foundation (to 570 K.T.), the Max Planck Society (to R.G.-O. and K.T.), a German Research 571 Foundation grant (SPP2125) (to R.G.-O. and K.T.), a predoctoral fellowship from 572 the Nakajima Foundation (to T.N.), and a Chinese Scholarship Council PhD 573 stipend (CSC Student ID 201808440401) (to Y.C.).

\section{Author contributions:}

575 T.N. and K.T. designed the research. T.N., Y.C., F.E., and Y.T. performed 576 experiments. T.N., E.D., and R.G.O. performed analysis. T.N. and K.T. wrote the 577 paper with input from all authors.

\section{Data availability:}

579 The RNA sequencing data used in this study are deposited in the National 580 Center for Biotechnology Information Gene Expression Omnibus database 581 (accession no. GSE150422). Key data and scripts are available at 582 https://github.com/tnobori/co-transcriptomics.

\section{Conflict of interest:}

584 The authors declare no conflict of interest. 


\section{Methods}

586 Plant materials and growth conditions

587 The Arabidopsis thaliana accession Col-0 plants were grown in a chamber at

$58822^{\circ} \mathrm{C}$ with a 10 -h light period and $60 \%$ relative humidity for 24 days and then in

589 another chamber at $22^{\circ} \mathrm{C}$ with a 12 -h light period and $60 \%$ relative humidity. For

590 all experiments, 31- to 33-day-old plants were used.

591 Bacterial strains

592 Commensal strains were previously isolated from wild $A$. thaliana plants (Bai et

593 al., 2015) (http://www.at-sphere.com/) (Table 1). The Pto mutant D36E was

594 previously described (Wei et al., 2015). Bacterial strains were cultured at $20^{\circ} \mathrm{C}$

595 (commensal strains) or $28^{\circ} \mathrm{C}$ (Pto and D36E) at $200 \mathrm{rpm}$ in liquid 50\% TSB

596 medium (Sigma-Aldrich, USA).

\section{Sampling of bacteria in vitro}

598 Commensal strains were grown in liquid 50\% TSB medium and harvested at the

599 late log phase. 0.1 volume of the stop buffer (95\% EtOH, 5\% Phenol) was added

600 to bacterial cultures before centrifuging to collect bacterial cells. Target $\mathrm{OD}_{600}$ for

601 each strain (the late log phase): Leaf1 $=0.7$, Leaf130 $=1.2$, Leaf155 $=0.5$,

602 Leaf176 $=0.9$, Leaf177 $=0.6$, Leaf187 $=0.8$, Leaf404 $=0.6$, Root935 $=0.8$,

603 Soil763 $=1.8$.

604 Bacterial inoculation to plant leaves and sampling

605 Commensal strains were grown in the liquid 50\% TSB medium. For each strain,

606 multiple cultures were prepared with different bacterial densities to ensure that

607 unsaturated cultures were used for experiments. Bacterial cells were harvested

608 by centrifugation, washed twice with sterile water, and resuspended in sterile 
609 water to $\mathrm{OD}_{600}$ of 0.5 . Plants grown in pots were randomized before bacterial

610 inoculation. For bacterial RNA-seq, 80-100 A. thaliana leaves (four fully

611 expanded leaves per plant) were syringe-inoculated with bacterial suspensions

612 using a needleless syringe. For plant RNA-seq, approximately six leaves (two

613 fully expanded leaves per plant) were treated. Mock control (water infiltration)

614 was included in every plant RNA-seq experiment. Leaves were harvested at 6

615 hours after inoculation. Sampling was conducted for one plant genotype at a

616 time, and approximately $5 \mathrm{~min}$ were needed per genotype. Leaves were

617 immediately frozen in liquid nitrogen and stored at $-80^{\circ} \mathrm{C}$. Three biological

618 replicates from independent experiments were taken for each condition of plant

619 and bacterial RNA-seq.

620 Sequencing library preparation and RNA sequencing

621 In planta bacterial transcriptome analysis was conducted as described previously

622 (Nobori et al., 2018) with slight modifications. Briefly, bacteria-infected leaves

623 were coarsely pulverized and released into bacterial isolation buffer $(9.5 \%$

624 ethanol, 0.5\% phenol, 25 mM TCEP (tris(2-carboxyethyl)phosphine) pH 4.5

625 adjusted with $\mathrm{NaOH}$ ) at $4^{\circ} \mathrm{C}$, filtered, and centrifuged to isolate bacterial cells

626 from plant cells. The original RNA extraction method based on chemical lysis of

627 bacterial cells by TriFast (Nobori et al., 2018) did not work for some bacterial

628 strains, thus we used FastRNA PRO ${ }^{\text {TM }}$ BLUE KIT (MP Biomedicals), which

629 involves mechanical cell lysis. rRNA was depleted to enrich mRNA, and the

630 cDNA libraries were prepared using Ovation Complete Prokaryotic RNA-seq kit

631 1-8 (NuGEN).

632 For plant RNA-seq, RNA was extracted with FastRNA PRO ${ }^{\text {TM }}$ KIT with Lysing

633 Matrix E (MP Biomedicals), and DNA was digested with TURBO DNase

634 (Ambion). RNA quality was determined using a 2100 Bioanalyzer (Agilent

635 Technologies, USA). Initially, $500 \mathrm{ng}$ total RNA was used for polyA enrichment

636 with the $\operatorname{NEBNext}{ }^{\circledR}$ Poly(A) mRNA Magnetic Isolation Module (New England 
637 Biolabs). Subsequent library preparation was performed with NEBNext Ultra $^{\mathrm{TM}}$

638 II Directional RNA Library Prep Kit for Illumina® (New England Biolabs)

639 according to the manufacturer's instructions.

640 Libraries were immobilized and processed onto a flow cell with cBot (Illumina)

641 and subsequently sequenced on the HiSeq3000 system (Illumina) with 1 x 150

642 bp single reads. Primary data analysis (incl. image analysis, cluster identification,

643 base calling, assignment of quality scores) has been performed with RTA

644 (real-time analysis software; Illumina) installed on the sequencing platform.

645 For bacterial and plant samples, approximately 10 and 30 million reads, 646 respectively, were obtained. Bacterial reads were mapped onto the

647 corresponding bacterial genomes (Bai et al., 2015) using Bowtie2 (Langmead 648 and Salzberg, 2012). Plant reads were mapped onto the Arabidopsis genome 649 (TAIR10) using HISAT2 (Kim et al., 2015). Mapped reads were counted with the 650 Python package HTSeq (Anders et al., 2015). The RNA-seq data used in this 651 study are deposited in NCBI Gene Expression Omnibus database (accession no. 652 GSE150422).

\section{Raw data}

654 Raw RNA-seq count and bacterial gene annotation files are available at 655 https://github.com/tnobori/co-transcriptomics.

\section{Data analysis - plant RNA-seq}

657 The statistical analysis of the RNA-seq data was performed in the R 658 environment. Genes with average counts $<5$ were excluded from the analysis. 659 The count data were TMM-normalized and log-transformed using the function 660 calcNormFactors in the package edgeR (Robinson et al., 2010) and the function 661 voomWithQualityWeights in the package limma (Ritchie et al., 2015), 662 respectively. To each gene, a linear model was fitted by using the function ImFit 663 in the limma package (Ritchie et al., 2015). The eBayes function in the limma 
664 package was used for variance shrinkage during the calculation of the p-values.

665 The false discovery rate (FDR; the Storey's q-values) was calculated using the

666 qvalue function in the qvalue package (Storey and Tibshirani, 2003). Genes with

667 q-value <0.01 and $\mid \log _{2}$ fold change| > 1 were defined as differentially expressed

668 genes. The prcomp function was used for principal component analysis.

669 Heatmaps were created with the pheatmap function in the $\mathrm{R}$ environment.

670 Enriched GO terms were identified using the BiNGO plugin for Cytoscape (Maere

671 et al., 2005). Scatter plots and box plots were generated using the R-package

672 ggplot2.

673 Data analysis - bacterial RNA-seq

674 Bacterial phylogenetic analysis

675 The bacterial genomes were searched for the bacterial small ribosomal subunit

676 16S rRNA gene using RNAmmer (Lagesen et al., 2007). Next, a multiple

677 sequence alignment was performed using Clustal Omega (Sievers et al., 2011)

678 with default parameters. Finally, we employed FastTree (Price et al., 2010) to

679 build a maximum-likelihood phylogeny based on the gamma time reversible 680 substitution model (GTR). This tree was visualized (Fig. 1B) using the interactive

681 Tree of Life (Letunic and Bork, 2019).

\section{Orthologous gene prediction and KEGG annotation}

683 De novo orthology prediction was performed by using OrthoFinder (Emms and 684 Kelly, 2015) with default parameters on the predicted protein coding sequences 685 from the bacterial genomes. Individual genes were annotated with the KEGG 686 database as a reference (Kanehisa et al., 2014) using the blastkoala webserver 687 (Prokaryotes group) (Kanehisa et al., 2016). Subsequently, orthologous genes 688 were assigned a single $\mathrm{KO}$ annotation by majority vote of individually annotated 689 sequences in each group. The genomes of the commensal strains were 
690 previously reported (Bai et al., 2015) and are available at our GitHub repository

691 (https://github.com/tnobori/co-transcriptomics).

\section{Data normalization and visualization}

693 RNA-seq data were normalized for each strain. After omitting genes with average 694 count < 5, count data was TMM-normalized and log-transformed as described 695 above. Genes with FDR <0.01 (corrected by Benjamini-Hochberg method) and $696 \log _{2}$ fold change| > 1 were defined as differentially expressed genes.

697 Commensal genes were annotated with OGs to integrate gene expression data 698 of different strains. When multiple genes are annotated with the same OG, the 699 mean expression value was taken. Data visualization was performed as 700 described above. UpSet plots were generated in the $\mathrm{R}$ environment using the 701 package UpSetR (Conway et al., 2017).

702 KO enrichment analysis (related to Fig. 2A and Fig. S3A)

703 A custom KEGG ontology (KO) database was created by taking only functional

704 terms encoded in at least one bacterial genome (downloaded in January 2019).

705 For each strain, a list of KOs was generated by subsetting the corresponding 706 KEGG IDs from the custom KO database (Data S6). KO enrichment test was 707 performed using a hypergeometric test (FDR corrected by Benjamini-Hochberg 708 method). KOs with FDR $<0.01$ and containing more than three genes were 709 defined as significantly enriched KOs. An R script and KO databases are 710 available at https://github.com/tnobori/co-transcriptomics.

711 Generating plots of genes with various functions (related to Fig. 2B-G, Fig. 4B-D 712 and F, Fig. S3B and C, Fig. S6, Fig. S7, Fig. S9-12)

713 Bacterial genes were selected by KEGG pathway annotations or keyword 714 searches from KEGG BRITE annotations. R scripts for this analysis are available 715 at https://github.com/tnobori/co-transcriptomics. 
716 Intersecting plant-associated bacterial genes and differentially regulated genes in

717 planta (related to Fig. 3)

718 In a previous study (Levy et al., 2017), comparative genomics analyses defined

719 "plant-associated (PA) genes" for each phylum/class using multiple statistical

720 tests. The study defined two groups of Actinobacteria (Actinobacteria1 and

721 Actinobacteria2). The Actinobacteria strains used in the present study are all

722 Actinobacteria1). We defined genes that passed at least one statistical test as

723 "PA genes" and the others were defined as nonPA genes. An R script and

724 PA-gene datasets for this analysis are available at

725 https://github.com/tnobori/co-transcriptomics.

726 MAMP conservation analysis (related to Fig. 5D)

727 Canonical flg22 and elf18 sequences were blasted against the bacterial

728 genomes using blastp (Camacho et al., 2009) with standard settings. The results

729 of these homology searches were filtered by retaining hits covering at least $90 \%$

730 of the length of the MAMP sequence in the alignment and subsequently

731 retrieving the alignment with the highest percentage identity.

\section{Integration of plant and bacterial RNA-seq data (related to Fig. 6)}

733 Co-transcriptome fold change data (bacteria: in planta vs. in vitro; plants: bacteria

734 vs. mock) of nine strains were used for this analysis. Plant genes whose

735 expression was significantly changed by at least one strain were used. Pearson's

736 correlation coefficients between individual plant genes and bacterial OGs were

737 calculated. The same analysis was performed for all the combinations of eight

738 strains (bootstrapping). Among these 8-strain and 9-strain datasets, the weakest

739 correlation coefficient value was used for each combination of a bacterial OG and

740 a plant gene (Fig. S8). An R script and plant/bacterial gene expression datasets

741 for this analysis are available at https://github.com/tnobori/co-transcriptomics. 


\section{Determination of bacterial colony forming units (related to Fig. S1)}

743 Bacterial colonization of the leaves was determined following a previous study

744 (Chen et al., 2020) with slight modifications. The $\mathrm{Cl}_{2}$-gas-sterilized seeds were

745 stratified for 2 days at $4^{\circ} \mathrm{C}$, sown on half Murashige \& Skoogs (MS,

746 Duchefa-Biochemie, MO255.0050) agar medium with 1\% sucrose, and allowed

747 to germinate for 5 days. Seedlings of the same physiological state were

748 transplanted on half MS agar medium and were grown for another 9 days (a total

749 of 2 weeks) prior to inoculation with bacteria. One day before inoculation,

750 bacterial cultures were grown on half TSB for 24 hours at $22^{\circ} \mathrm{C}$ with $200 \mathrm{rpm}$

751 shaking. On the day of inoculation, bacterial cells were harvested by

752 centrifugation at 3000 rpm for 15 min, washed twice with sterile water, and then

753 finally suspended in $10 \mathrm{mM} \mathrm{MgCl}_{2}$. The resulting bacterial suspensions were

754 diluted to a final $O D_{600}$ of 0.5 with sterile water and with this, each plate of

755 2-week-old seedlings was flood-inoculated for $1 \mathrm{~min}$, drained, and allowed to dry

756 for $15 \mathrm{~min}$. Plants were then grown for 3 days and 2-3 leaves of the same

757 physiological state were harvested aseptically and weighed. To quantify bacteria

758 in the endophytic compartment, leaves were surface-sterilized with $75 \%$ ethanol

759 for 30 seconds and washed twice with sterile water, and the leaves were

760 homogenized on $10 \mathrm{mM} \mathrm{MgCl}^{2}$ buffer using TissueLyserll (Qiagen) with the

761 frequency of $30 \mathrm{~s}^{-1}$ for $5 \mathrm{~min}$. The samples were then serially diluted $\left(10^{0}\right.$ to $\left.10^{5}\right)$

762 and spread-plated on 0.5x TSB agar medium. Plates were incubated at ambient

763 temperature, colonies were observed and counted for 1-3 d and colony forming

764 units were expressed per mg FW. The total compartment was assayed similarly

765 but without surface sterilization. 
bioRxiv preprint doi: https://doi.org/10.1101/2021.04.25.440543; this version posted April 26, 2021. The copyright holder for this preprint (which

was not certified by peer review) is the author/funder, who has granted bioRxiv a license to display the preprint in perpetuity. It is made available under ACC-BY-NC-ND 4.0 International license.

766 Data S1 OG distribution

767 Data S2 Expression of all OGs

768 Data S3 List of PA and nonPA KOs

769 Data S4 Plant RNA-seq data

770 Data S5 Correlation matrix of plant and bacterial transcriptomes

771 Data S6 KO database for each strain 


\section{References}

773 Anders, S., Pyl, P.T., and Huber, W. (2015). HTSeq-a Python framework to work with 774 high-throughput sequencing data. Bioinformatics 31, 166-169.

775 Bai, Y., Müller, D.B., Srinivas, G., Garrido-Oter, R., Potthoff, E., Rott, M., Dombrowski, 776 N., Münch, P.C., Spaepen, S., Remus-Emsermann, M., et al. (2015). Functional overlap 777 of the Arabidopsis leaf and root microbiota. Nature 528, 364-369.

778 Boller, T., and Felix, G. (2009). A renaissance of elicitors: perception of 779 microbe-associated molecular patterns and danger signals by pattern-recognition 780 receptors. Annu. Rev. Plant Biol. 60, 379-406.

781 Camacho, C., Coulouris, G., Avagyan, V., Ma, N., Papadopoulos, J., Bealer, K., and 782 Madden, T.L. (2009). BLAST+: architecture and applications. BMC Bioinformatics 10, 783421.

784 Carrión, V.J., Perez-Jaramillo, J., Cordovez, V., Tracanna, V., de Hollander, M., 785 Ruiz-Buck, D., Mendes, L.W., van ljcken, W.F.J., Gomez-Exposito, R., Elsayed, S.S., et 786 al. (2019). Pathogen-induced activation of disease-suppressive functions in the 787 endophytic root microbiome. Science 366, 606-612.

788 Chapelle, E., Alunni, B., Malfatti, P., Solier, L., Pédron, J., Kraepiel, Y., and Van 789 Gijsegem, F. (2015). A straightforward and reliable method for bacterial in planta 790 transcriptomics: application to the Dickeya dadantii/Arabidopsis thaliana pathosystem. 791 Plant J. 82, 352-362.

792 Chen, L.-Q., Hou, B.-H., Lalonde, S., Takanaga, H., Hartung, M.L., Qu, X.-Q., Guo, 793 W.-J., Kim, J.-G., Underwood, W., Chaudhuri, B., et al. (2010). Sugar transporters for 794 intercellular exchange and nutrition of pathogens. Nature 468, 527-532.

795 Chen, T., Nomura, K., Wang, X., Sohrabi, R., Xu, J., Yao, L., Paasch, B.C., Ma, L., 796 Kremer, J., Cheng, Y., et al. (2020). A plant genetic network for preventing dysbiosis in 797 the phyllosphere. Nature 580, 653-657.

798 Conway, J.R., Lex, A., and Gehlenborg, N. (2017). UpSetR: an R package for the 799 visualization of intersecting sets and their properties. Bioinformatics 33, 2938-2940.

800 Costa, T.R.D., Felisberto-Rodrigues, C., Meir, A., Prevost, M.S., Redzej, A., Trokter, M., 801 and Waksman, G. (2015). Secretion systems in Gram-negative bacteria: structural and 802 mechanistic insights. Nat. Rev. Microbiol. 13, 343-359.

803 Durán, P., Thiergart, T., Garrido-Oter, R., Agler, M., Kemen, E., Schulze-Lefert, P., and 804 Hacquard, S. (2018). Microbial Interkingdom Interactions in Roots Promote Arabidopsis 805 Survival. Cell 175, 973-983.e14.

806 Emms, D.M., and Kelly, S. (2015). OrthoFinder: solving fundamental biases in whole 807 genome comparisons dramatically improves orthogroup inference accuracy. Genome 
Biol. 16, 1-14.

809 Felix, G., Duran, J.D., Volko, S., and Boller, T. (1999). Plants have a sensitive perception 810 system for the most conserved domain of bacterial flagellin. Plant J. 18, 265-276.

811 Garrido-Oter, R., Nakano, R.T., Dombrowski, N., Ma, K.-W., AgBiome Team, McHardy, 812 A.C., and Schulze-Lefert, P. (2018). Modular Traits of the Rhizobiales Root Microbiota 813 and Their Evolutionary Relationship with Symbiotic Rhizobia. Cell Host Microbe 24, 814 155-167.e5.

815 Hacquard, S., Garrido-Oter, R., González, A., Spaepen, S., Ackermann, G., Lebeis, S., 816 McHardy, A.C., Dangl, J.L., Knight, R., Ley, R., et al. (2015). Microbiota and Host 817 Nutrition across Plant and Animal Kingdoms. Cell Host \& Microbe 17, 603-616.

818 Hacquard, S., Spaepen, S., Garrido-Oter, R., and Schulze-Lefert, P. (2017). Interplay 819 Between Innate Immunity and the Plant Microbiota. Annu. Rev. Phytopathol. 55, 820 565-589.

821 Helmann, T.C., Deutschbauer, A.M., and Lindow, S.E. (2019). Genome-wide 822 identification of Pseudomonas syringae genes required for fitness during colonization of 823 the leaf surface and apoplast. Proc. Natl. Acad. Sci. U. S. A. 116, 18900-18910.

824 Hillmer, R.A., Tsuda, K., Rallapalli, G., Asai, S., Truman, W., Papke, M.D., Sakakibara, 825 H., Jones, J.D.G., Myers, C.L., and Katagiri, F. (2017). The highly buffered Arabidopsis 826 immune signaling network conceals the functions of its components. PLOS Genetics 13, 827 e1006639.

828 Jones, J.D.G., and Dangl, J.L. (2006). The plant immune system. Nature 444, 323-329.

829 Kanehisa, M., Goto, S., Sato, Y., Kawashima, M., Furumichi, M., and Tanabe, M. (2014). 830 Data, information, knowledge and principle: back to metabolism in KEGG. Nucleic Acids 831 Res. 42, D199-D205.

832 Kanehisa, M., Sato, Y., and Morishima, K. (2016). BlastKOALA and GhostKOALA: 833 KEGG Tools for Functional Characterization of Genome and Metagenome Sequences. 834 J. Mol. Biol. 428, 726-731.

835 Kim, D., Langmead, B., and Salzberg, S.L. (2015). HISAT: a fast spliced aligner with low 836 memory requirements. Nat. Methods 12, 357-360.

837 Kunze, G., Zipfel, C., Robatzek, S., Niehaus, K., Boller, T., and Felix, G. (2004). The N 838 terminus of bacterial elongation factor Tu elicits innate immunity in Arabidopsis plants. 839 Plant Cell 16, 3496-3507.

840 Kwak, M.-J., Kong, H.G., Choi, K., Kwon, S.-K., Song, J.Y., Lee, J., Lee, P.A., Choi, S.Y., 841 Seo, M., Lee, H.J., et al. (2018). Rhizosphere microbiome structure alters to enable wilt 842 resistance in tomato. Nat. Biotechnol.

843 Lagesen, K., Hallin, P., Rødland, E.A., Staerfeldt, H.-H., Rognes, T., and Ussery, D.W. 844 (2007). RNAmmer: consistent and rapid annotation of ribosomal RNA genes. Nucleic 
845 Acids Res. 35, 3100-3108.

846 Langmead, B., and Salzberg, S.L. (2012). Fast gapped-read alignment with Bowtie 2. 847 Nat. Methods 9, 357-359.

848 Lebeis, S.L., Paredes, S.H., Lundberg, D.S., Breakfield, N., Gehring, J., McDonald, M., 849 Malfatti, S., del Rio, T.G., Jones, C.D., Tringe, S.G., et al. (2015). Salicylic acid 850 modulates colonization of the root microbiome by specific bacterial taxa. Science 349 , $851860-864$.

852 Letunic, I., and Bork, P. (2019). Interactive Tree Of Life (iTOL) v4: recent updates and 853 new developments. Nucleic Acids Res. 47, W256-W259.

854 Levy, A., Salas Gonzalez, I., Mittelviefhaus, M., Clingenpeel, S., Herrera Paredes, S., 855 Miao, J., Wang, K., Devescovi, G., Stillman, K., Monteiro, F., et al. (2017). Genomic 856 features of bacterial adaptation to plants. Nat. Genet. 50, 138-150.

857 Levy, A., Conway, J.M., Dangl, J.L., and Woyke, T. (2018). Elucidating Bacterial Gene 858 Functions in the Plant Microbiome. Cell Host Microbe 24, 475-485.

859 Lovelace, A.H., Smith, A., and Kvitko, B.H. (2018). Pattern-Triggered Immunity Alters the 860 Transcriptional Regulation of Virulence-Associated Genes and Induces the Sulfur 861 Starvation Response in Pseudomonas syringae pv. tomato DC3000. Mol. Plant. 862 Microbe. Interact. 31, 750-765.

863 Maere, S., Heymans, K., and Kuiper, M. (2005). BiNGO: a Cytoscape plugin to assess 864 overrepresentation of gene ontology categories in biological networks. Bioinformatics 21, 865 3448-3449.

866 Müller, D.B., Schubert, O.T., Röst, H., Aebersold, R., and Vorholt, J.A. (2016). 867 Systems-level Proteomics of Two Ubiquitous Leaf Commensals Reveals 868 Complementary Adaptive Traits for Phyllosphere Colonization. Mol. Cell. Proteomics 15, 869 3256-3269.

870 Nobori, T., Velásquez, A.C., Wu, J., Kvitko, B.H., Kremer, J.M., Wang, Y., He, S.Y., and 871 Tsuda, K. (2018). Transcriptome landscape of a bacterial pathogen under plant 872 immunity. Proc. Natl. Acad. Sci. U. S. A. 115, E3055-E3064.

873 Nobori, T., Wang, Y., Wu, J., Stolze, S.C., and Tsuda, Y. (2019). In planta bacterial 874 multi-omics analysis illuminates regulatory principles underlying plant-pathogen 875 interactions. BioRxiv.

876 Nobori, T., Wang, Y., Wu, J., Stolze, S.C., Tsuda, Y., Finkemeier, I., Nakagami, H., and 877 Tsuda, K. (2020). Multidimensional gene regulatory landscape of a bacterial pathogen in 878 plants. Nat Plants 6, 883-896.

879 Osborne, A.R., Rapoport, T.A., and van den Berg, B. (2005). Protein translocation by the 880 Sec61/SecY channel. Annu. Rev. Cell Dev. Biol. 21, 529-550.

881 Price, M.N., Dehal, P.S., and Arkin, A.P. (2010). FastTree 2--approximately 
882 maximum-likelihood trees for large alignments. PLoS One 5.

883 Ritchie, M.E., Phipson, B., Wu, D., Hu, Y., Law, C.W., Shi, W., and Smyth, G.K. (2015).

884 limma powers differential expression analyses for RNA-sequencing and microarray 885 studies. Nucleic Acids Res. 43, e47.

886 Robinson, M.D., McCarthy, D.J., and Smyth, G.K. (2010). edgeR: a Bioconductor 887 package for differential expression analysis of digital gene expression data. 888 Bioinformatics 26, 139-140.

889 Russell, A.B., Peterson, S.B., and Mougous, J.D. (2014). Type VI secretion system 890 effectors: poisons with a purpose. Nat. Rev. Microbiol. 12, 137-148.

891 Sievers, F., Wilm, A., Dineen, D., Gibson, T.J., Karplus, K., Li, W., Lopez, R., McWilliam, 892 H., Remmert, M., Söding, J., et al. (2011). Fast, scalable generation of high-quality 893 protein multiple sequence alignments using Clustal Omega. Mol. Syst. Biol. 7.

894 Storey, J.D., and Tibshirani, R. (2003). Statistical significance for genomewide studies. 895 Proc. Natl. Acad. Sci. U. S. A. 100, 9440-9445.

896 Teixeira, P.J.P.L., Teixeira, P.J.P., Colaianni, N.R., Law, T.F., Conway, J.M., Gilbert, S., Li, 897 H., Salas-González, I., Panda, D., Del Risco, N.M., et al. (2021). Specific modulation of 898 the root immune system by a community of commensal bacteria. Proceedings of the 899 National Academy of Sciences 118, e2100678118.

900 Toruño, T.Y., Stergiopoulos, I., and Coaker, G. (2016). Plant-Pathogen Effectors: Cellular 901 Probes Interfering with Plant Defenses in Spatial and Temporal Manners. Annu. Rev. 902 Phytopathol. 54, 419-441.

903 Vogel, C., Bodenhausen, N., Gruissem, W., and Vorholt, J.A. (2016). The Arabidopsis 904 leaf transcriptome reveals distinct but also overlapping responses to colonization by 905 phyllosphere commensals and pathogen infection with impact on plant health. New 906 Phytologist 212, 192-207.

907 Wei, H.-L., Chakravarthy, S., Mathieu, J., Helmann, T.C., Stodghill, P., Swingle, B., 908 Martin, G.B., and Collmer, A. (2015). Pseudomonas syringae pv. tomato DC3000 Type 909 III Secretion Effector Polymutants Reveal an Interplay between HopAD1 and AvrPtoB. 910 Cell Host \& Microbe 17, 752-762.

911 Xin, X.-F., Nomura, K., Aung, K., Velásquez, A.C., Yao, J., Boutrot, F., Chang, J.H., 912 Zipfel, C., and He, S.Y. (2016). Bacteria establish an aqueous living space in plants 913 crucial for virulence. Nature 539, 524-529.

914 Yamada, K., Saijo, Y., Nakagami, H., and Takano, Y. (2016). Regulation of sugar 915 transporter activity for antibacterial defense in Arabidopsis. Science 354, 1427-1430.

916 Young, G.M., Amid, D., and Miller, V.L. (1996). A bifunctional urease enhances survival 917 of pathogenic Yersinia enterocolitica and Morganella morganii at low pH. J. Bacteriol. 918 178, 6487-6495. 
919 Yu, K., Liu, Y., Tichelaar, R., Savant, N., Lagendijk, E., van Kuijk, S.J.L., Stringlis, I.A., 920 van Dijken, A.J.H., Pieterse, C.M.J., Bakker, P.A.H.M., et al. (2019).

921 Rhizosphere-Associated Pseudomonas Suppress Local Root Immune Responses by

922 Gluconic Acid-Mediated Lowering of Environmental pH. Curr. Biol. 29, 3913-3920.e4.

923 Yu, X., Lund, S.P., Scott, R. a., Greenwald, J.W., Records, A.H., Nettleton, D., Lindow, 924 S.E., Gross, D.C., and Beattie, G. a. (2013). Transcriptional responses of Pseudomonas 925 syringae to growth in epiphytic versus apoplastic leaf sites. Proc. Natl. Acad. Sci. U. S. 926 A. 110, E425-E434.

927 Yu, X., Lund, S.P., Greenwald, J.W., Records, A.H., Scott, R.A., Nettleton, D., Lindow, 928 S.E., Gross, D.C., and Beattie, A. (2014). Transcriptional Analysis of the Global 929 Regulatory Networks Active in Pseudomonas syringae during Leaf Colonization. MBio 5, 930 01614-e01683. 


\section{Supplementary figures}

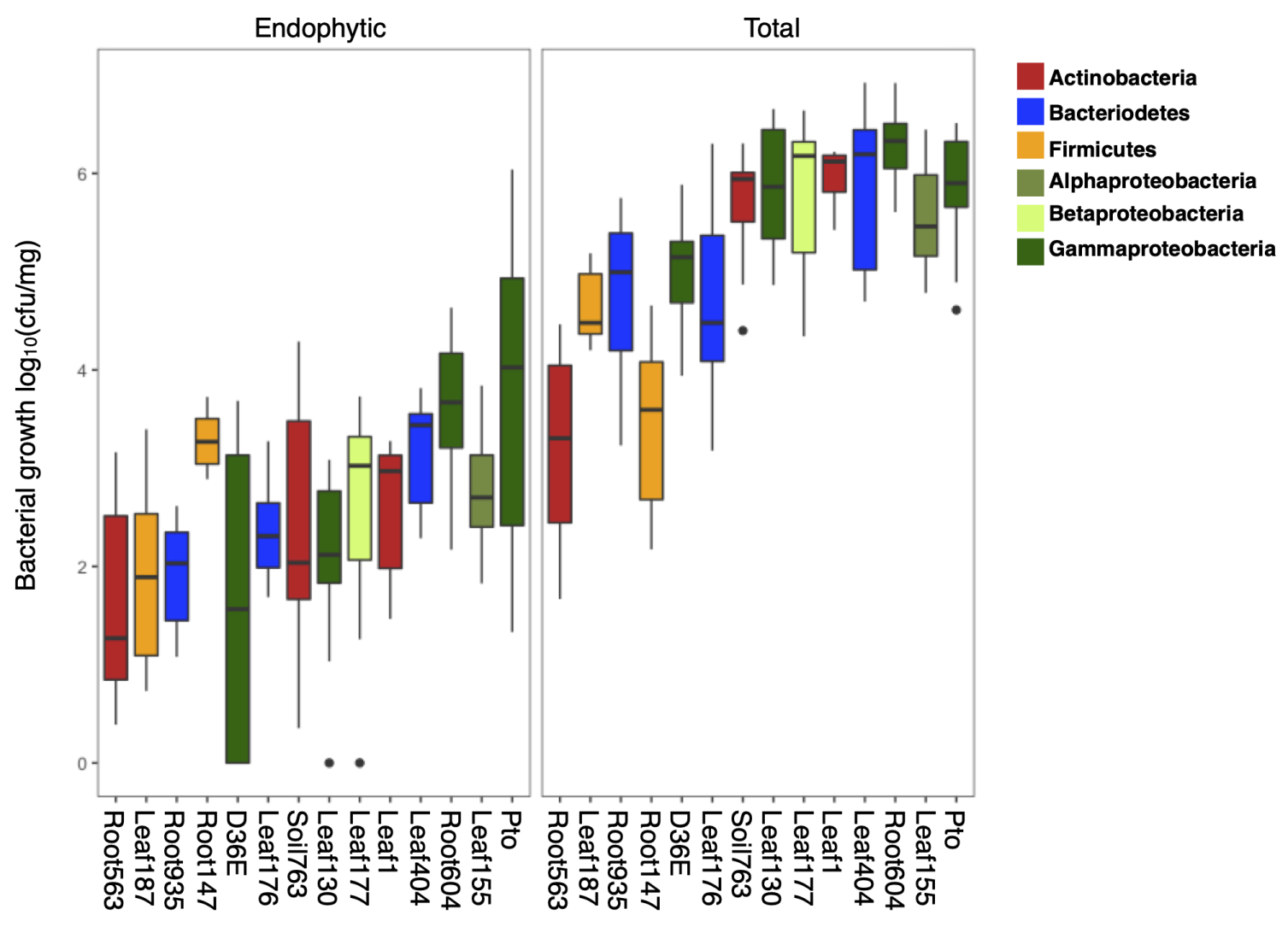

932 Fig. S1 Bacterial growth in plant leaves Bacteria were flood-inoculated to 933 three-week-old $A$. thaliana Col-0 at $\mathrm{OD}_{600}=0.5$. Bacterial growth was measured 934 three days after inoculation. Endophytic bacteria were counted after washing and 935 sterilizing the leaf surface, whereas total bacteria were counted without any 936 surface washing and sterilization (see Methods). 
A

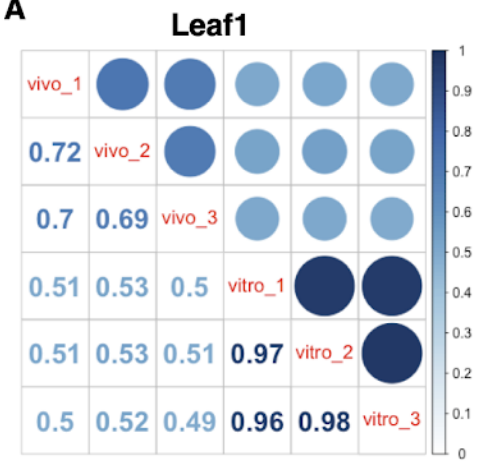

Leaf176

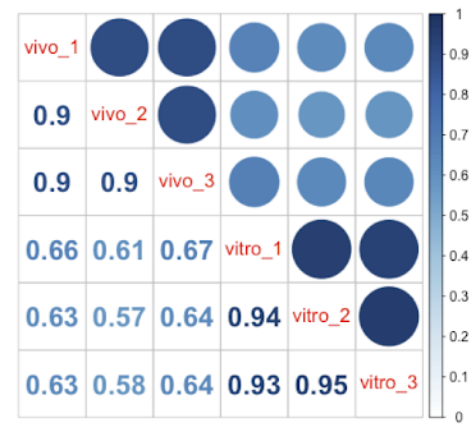

937

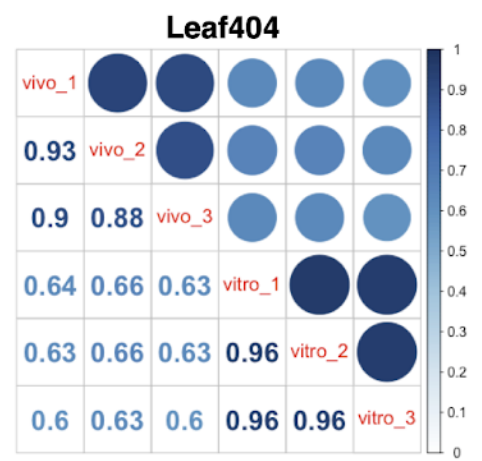

Leaf130

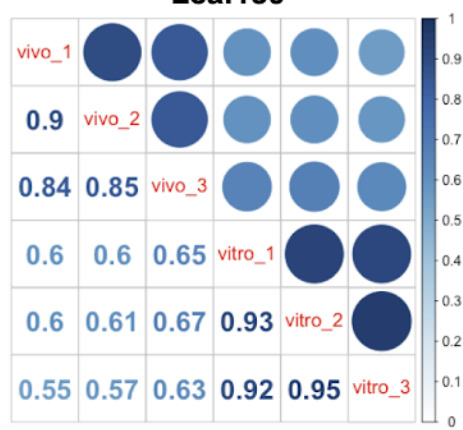

Leaf177

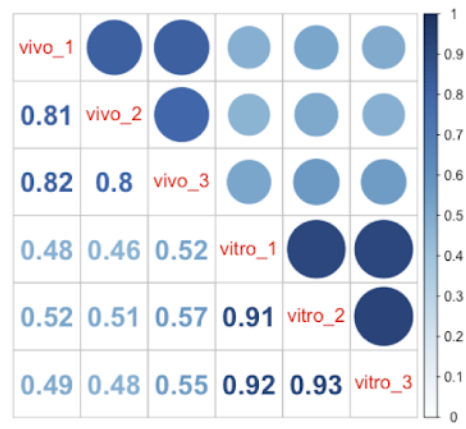

Root935

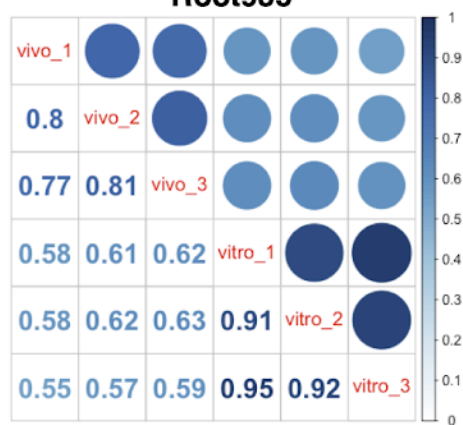

Leaf155

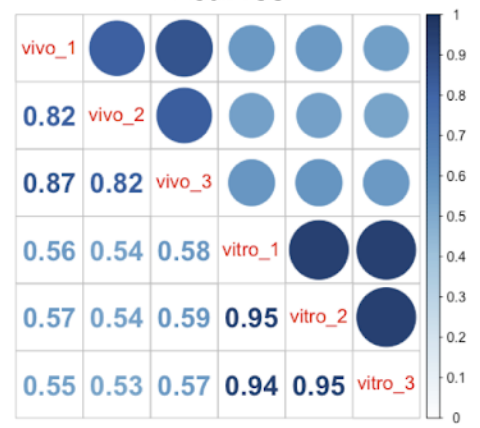

\section{Leaf187}

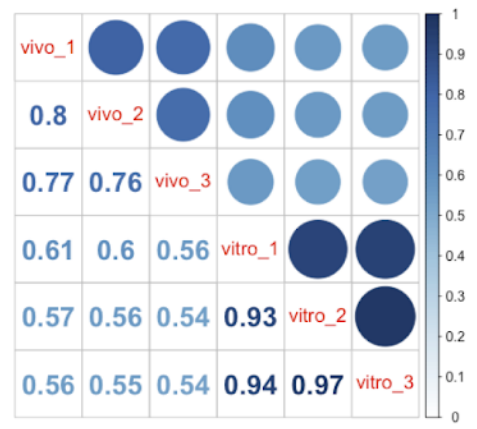

Soil763

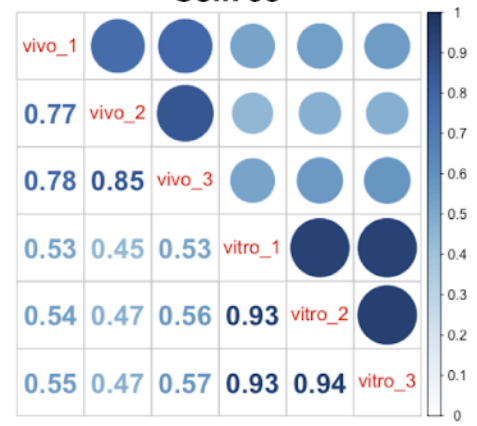

B

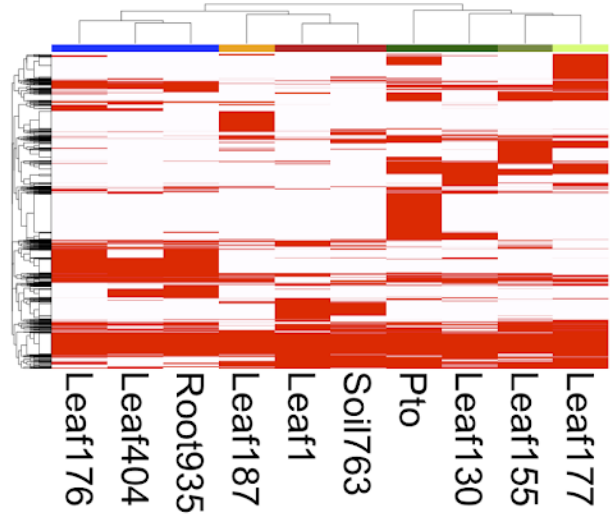

Actinobacteria

Bacteriodetes

Firmicutes

Alphaproteobacteria

Betaproteobacteria

Gammaproteobacteria

938 Fig. $\mathbf{S 2}$ Transcriptome analysis of bacteria (A) The correlation plot of each 939 replicate of bacterial RNA-seq data for individual strains. vivo: bacteria in plants. 940 vitro: bacteria in rich media. (B) Genes that exist are colored in red. The 
bioRxiv preprint doi: https://doi.org/10.1101/2021.04.25.440543; this version posted April 26, 2021. The copyright holder for this preprint (which

was not certified by peer review) is the author/funder, who has granted bioRxiv a license to display the preprint in perpetuity. It is made available under aCC-BY-NC-ND 4.0 International license.

941 taxonomic affiliation (phylum/class level) of each strain is indicated with different 942 colors. See Data S1 for the gene presence-absence table. 
A
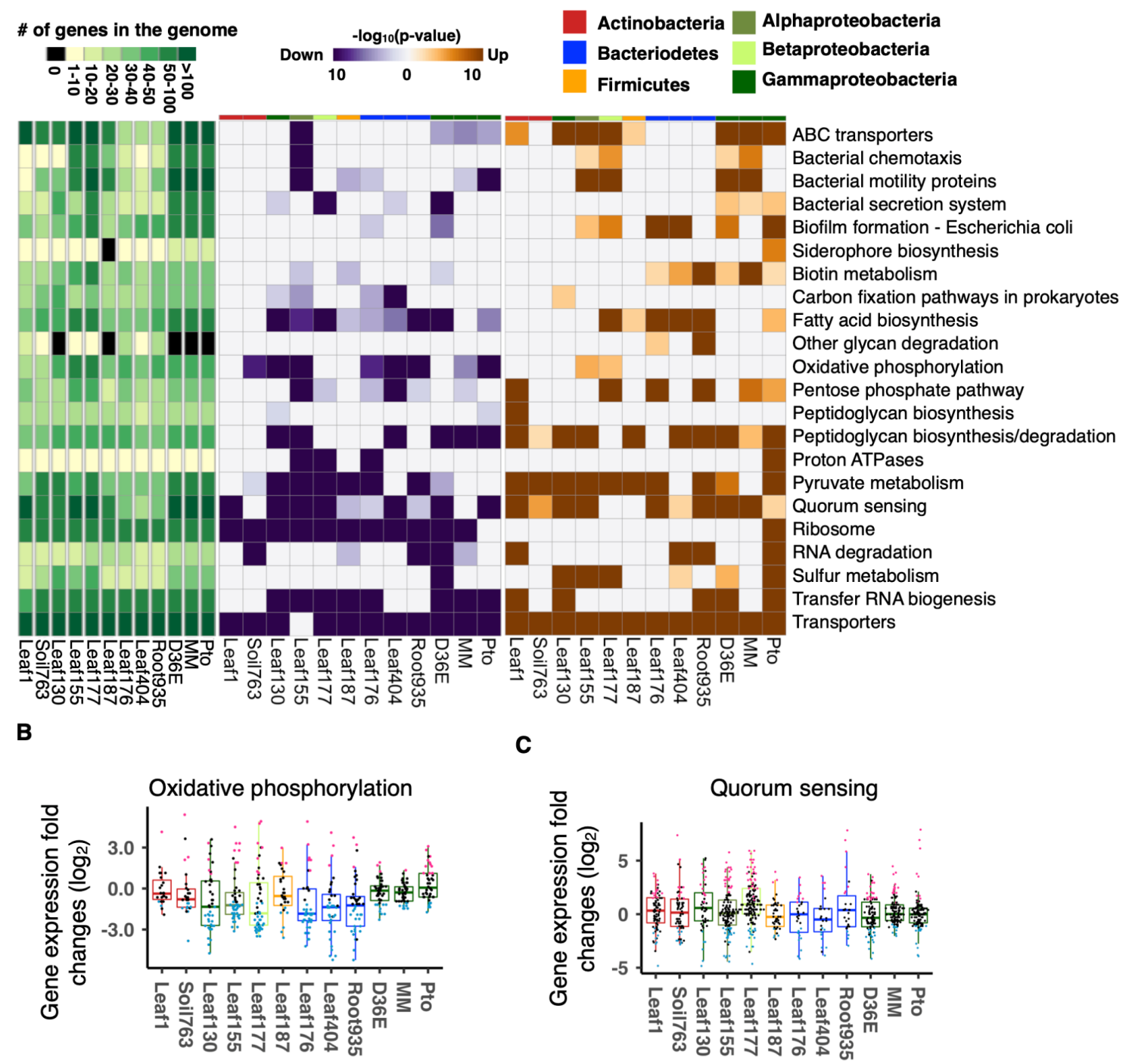

943 Fig. S3: Conserved and strain-specific regulation of bacterial functions in 944 plants (A) KEGG ontology (KO) terms enriched in genes that are significantly 945 up- (orange) or down (purple)-regulated in planta compared with in vitro (rich 946 media). The heatmaps indicate $-\log _{10}$ p-values (FDR corrected by 947 Benjamini-Hochberg method). The top color bars indicate the taxonomic 948 affiliation (phylum/class level) of each strain. (B and C) Expression fold changes 949 (in planta vs. in vitro) of genes involved in (B) Oxidative phosphorylation and (C) 950 Quorum sensing. MM, Pto grown in a minimal medium. Results are shown as 951 box plots with boxes displaying the 25th-75th percentiles, the centerline 952 indicating the median, whiskers extending to the minimum, and maximum values 953 no further than 1.5 inter-quartile range. Box color indicates the taxonomic 954 affiliation (phylum/class level) of each strain. All individual data points (genes) are 955 overlaid with colors for DEGs (red: upregulated, blue: downregulated, black: 956 non-DEG). 


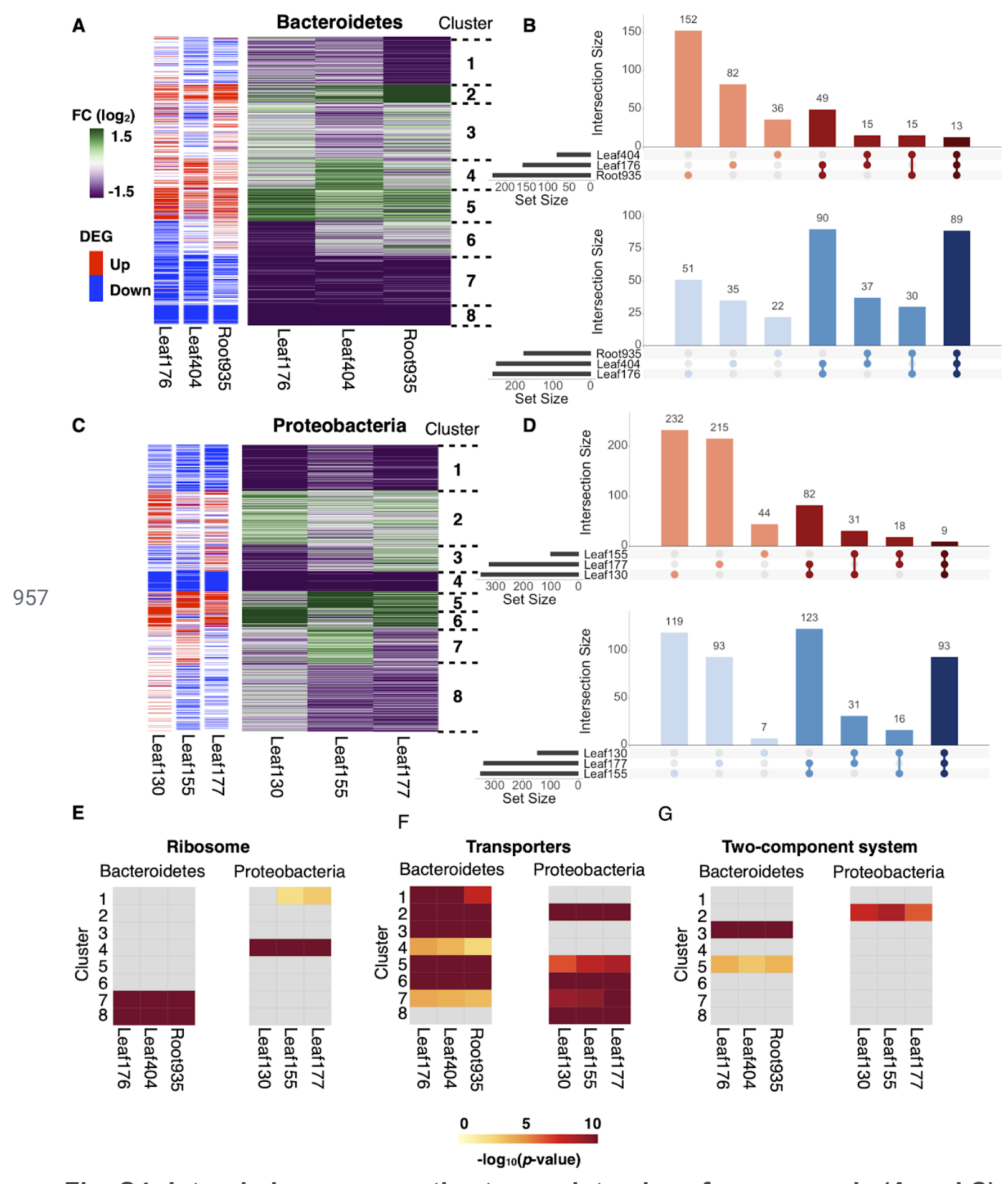

958 Fig. S4: Intraphylum comparative transcriptomics of commensals (A and C)

959 Gene expression fold changes between in planta and in vitro (rich media) of (A)

960 Bacteroidetes and (C) Proteobacteria strains. Orthologous groups shared among

961 three strains were used for the analysis. Differentially expressed genes (DEGs)

962 (in planta vs. in vitro; $\left|\log _{2} \mathrm{FC}\right|>1$; FDR $<0.01$; two-tailed Student's t test

963 followed by Storey's q-value) are indicated in the sidebars. Gene clusters defined

964 by k-mean clustering are shown $(k=8)$. ( $B$ and $D)$ UpSet intersection plots of 
bioRxiv preprint doi: https://doi.org/10.1101/2021.04.25.440543; this version posted April 26, 2021. The copyright holder for this preprint (which

was not certified by peer review) is the author/funder, who has granted bioRxiv a license to display the preprint in perpetuity. It is made available under aCC-BY-NC-ND 4.0 International license.

965 DEGs either up- (red) or down (blue)-regulated in planta in the (B) Bacteroidetes 966 and (D) Proteobacteria strains. Intersection size and set size indicate the number 967 of shared DEGs and the number of DEGs in each strain, respectively. (E-G) 968 Enrichment analysis of genes with the KEGG ontology terms (E) "Ribosome", (F) 969 "Transporters", and (G) "Two-component system". 


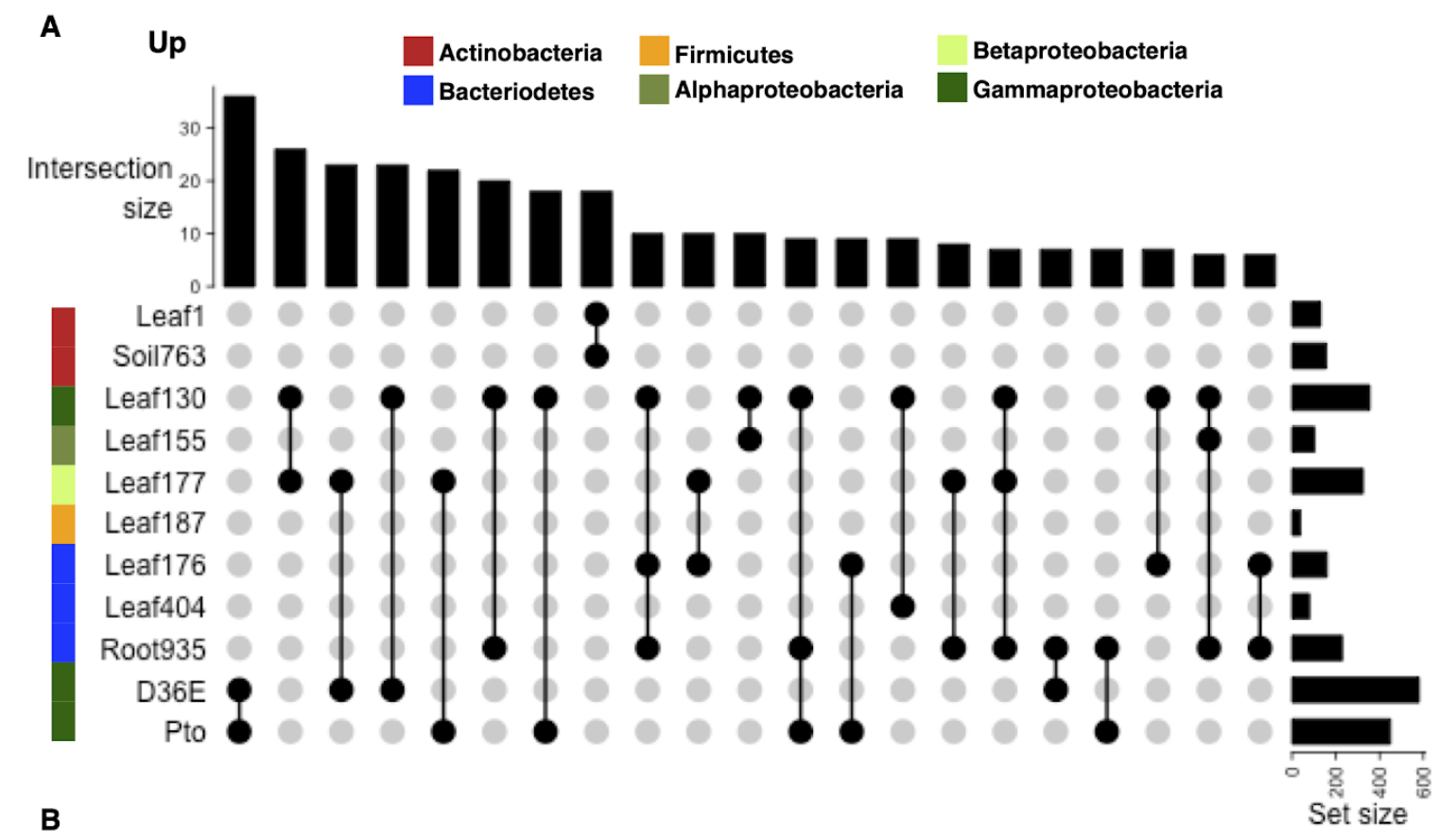

Down

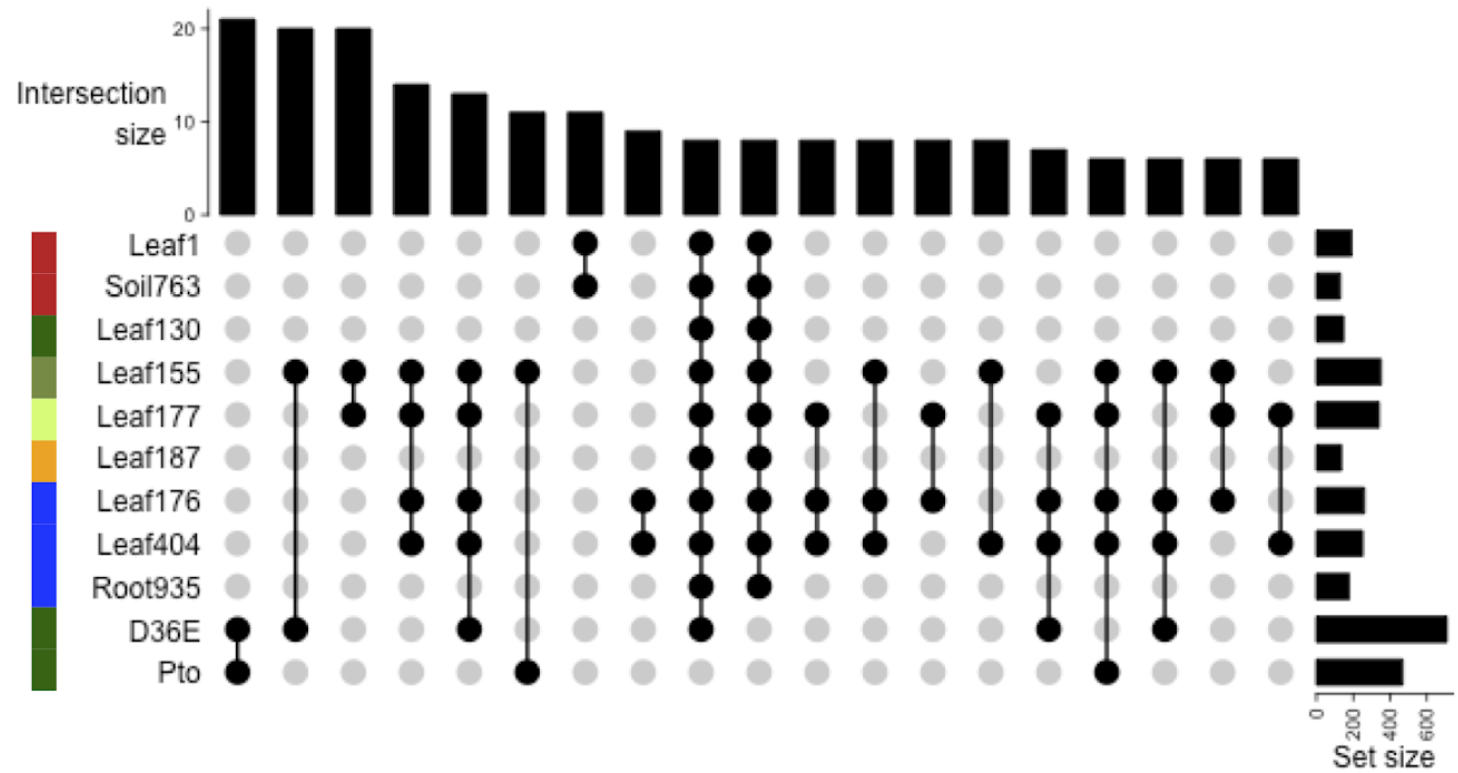

970 Fig. S5: Bacterial genes differentially regulated in plants UpSet intersection 971 plots of differentially expressed genes (DEGs; $\left|\log _{2} \mathrm{FC}\right|>1$; FDR $<0.01$; 972 two-tailed Student's $t$ test followed by Storey's q-value) either (A) up- or (B) 973 downregulated in planta. Intersection size and set size indicate the number of 974 shared DEGs and the number of DEGs in each strain, respectively. 975 Combinations of more than one strain with intersection size $>5$ are shown. The 976 color sidebars indicate the taxonomic affiliation. 


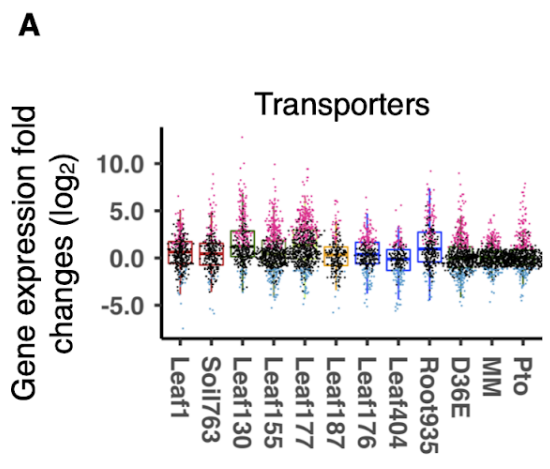

$\mathbf{B}$

977
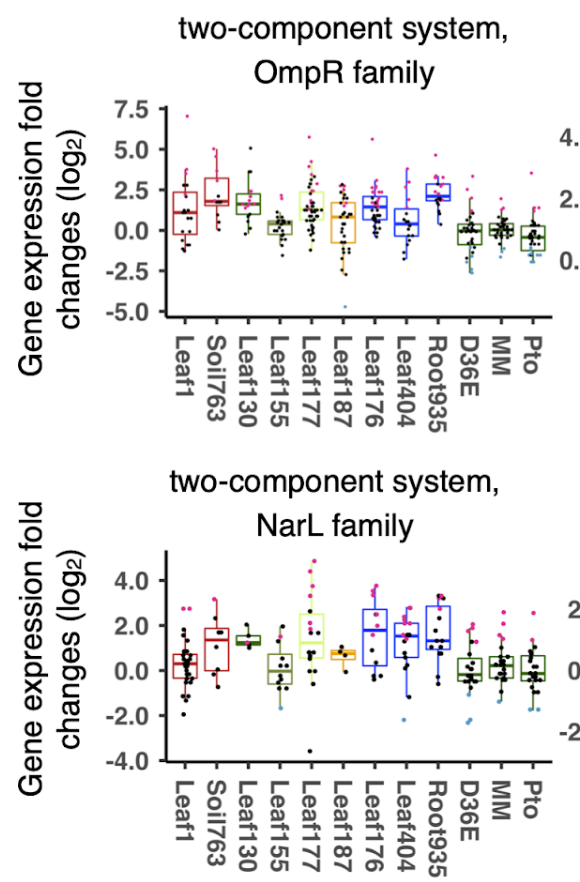

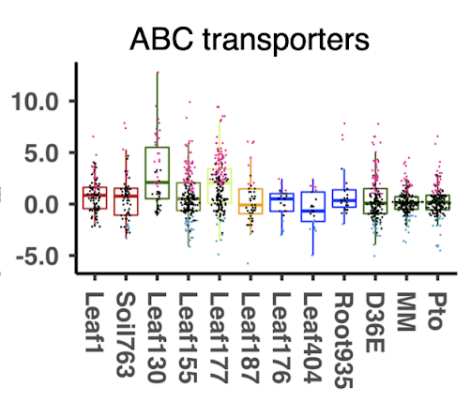

two-component system, LytR family

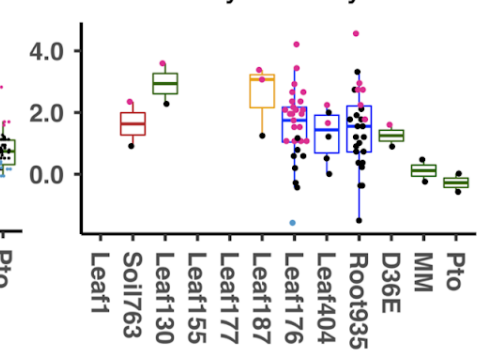

two-component system, CitB family
Actinobacteria

Alphaproteobacteria

Betaproteobacteria

Gammaproteobacteria

Firmicutes

Bacteriodetes two-component system, NtrC family

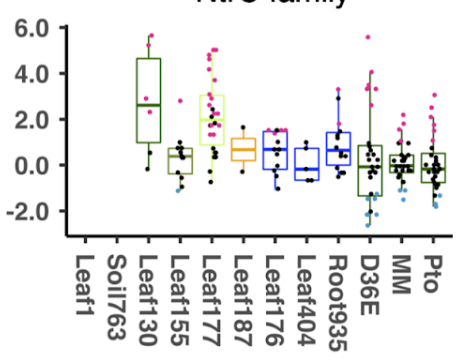

978 Fig. S6: Expression of various processes of commensals in planta ( $A$ and

979 B) Expression fold changes (in planta vs. in vitro) of genes related to (A) 980 transporters and (B) two-component system. Results are shown as box plots with 981 boxes displaying the 25th-75th percentiles, the centerline indicating the median, 982 whiskers extending to the minimum, and maximum values no further than 1.5 983 inter-quartile range. Box color indicates the taxonomic affiliation (phylum/class 984 level) of each strain. All individual data points (genes) are overlaid with colors for 985 DEGs (red: upregulated, blue: downregulated, black: non-DEG). 

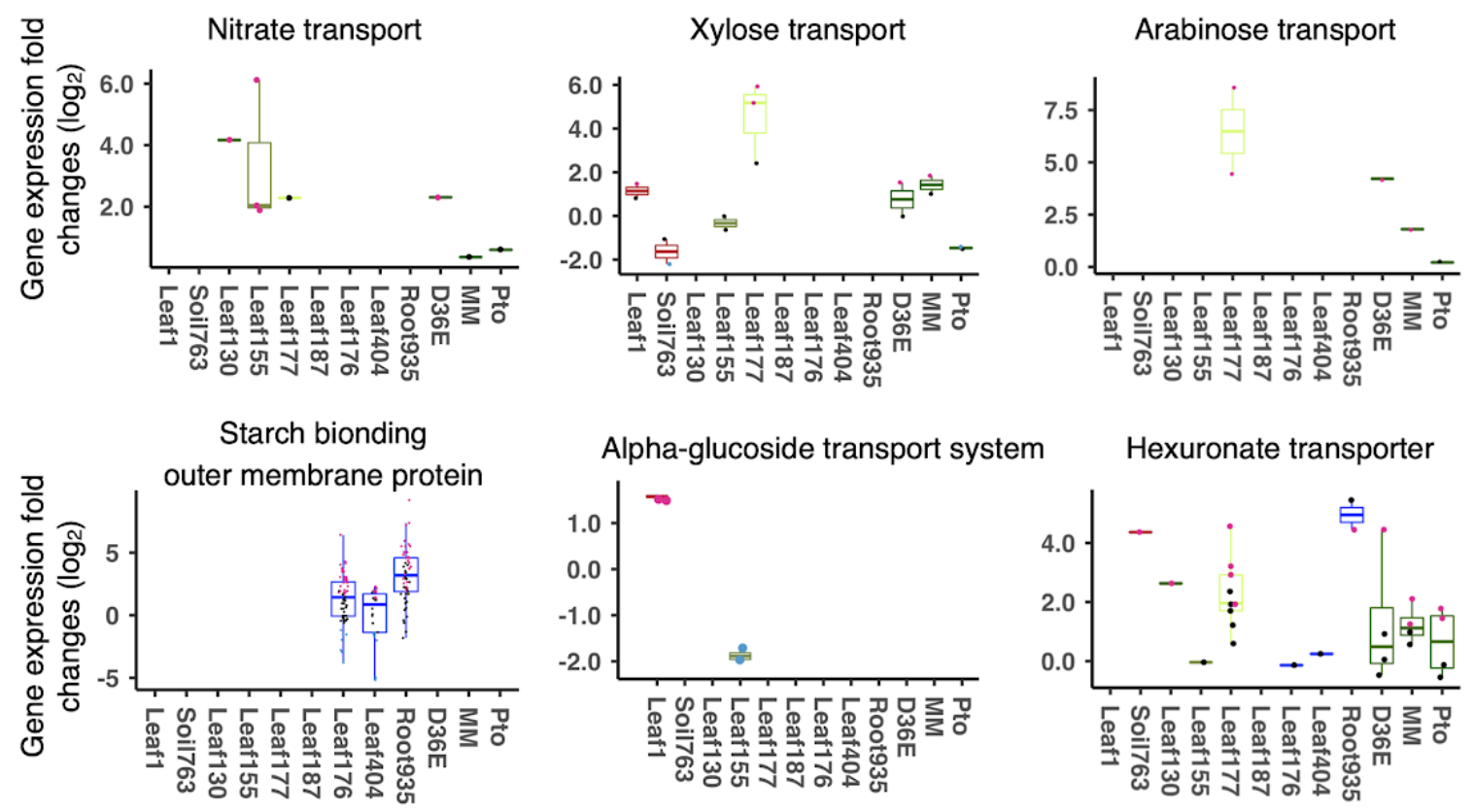

Hexuronate transporter
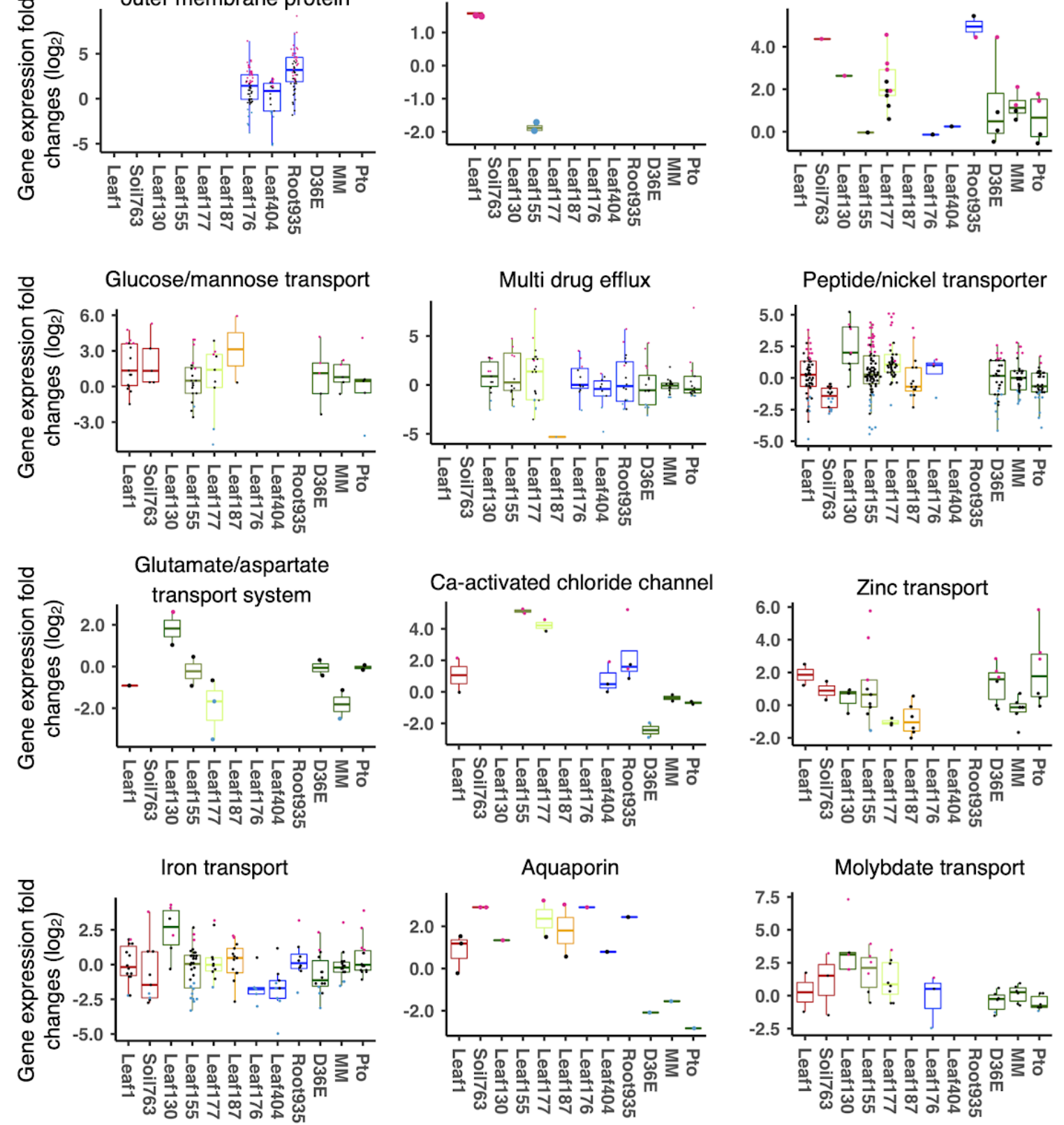

987 Fig. S7: Expression of genes related to nutrient acquisition processes in 988 commensals Expression fold changes (in planta vs. in vitro) of genes related to 
989 nutrient transporters. Results are shown as box plots with boxes displaying the 990 25th-75th percentiles, the centerline indicating the median, whiskers extending 991 to the minimum, and maximum values no further than 1.5 inter-quartile range. 992 Box color indicates the taxonomic affiliation (phylum/class level) of each strain 993 (see Fig. S6 for the color code). All individual data points (genes) are overlaid 994 with colors for DEGs (red: upregulated, blue: downregulated, black: non-DEG). 


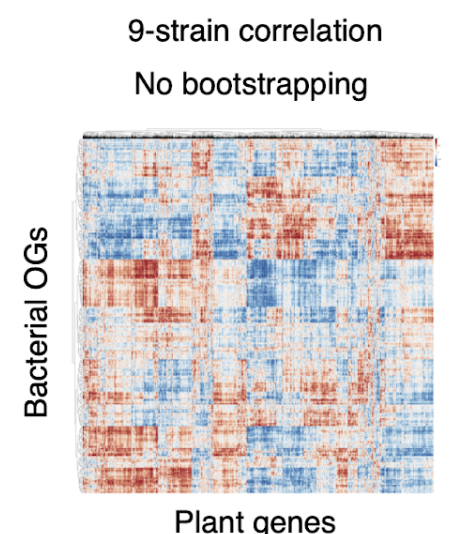

\section{8-strain correlation}

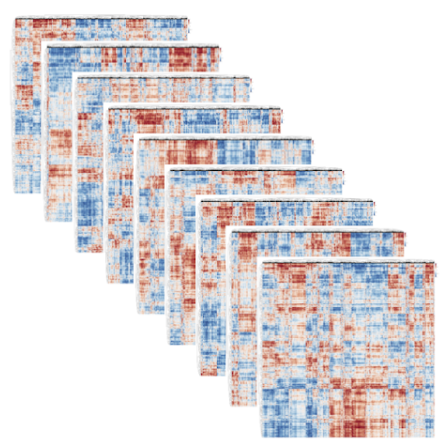

9-strain correlation

After bootstrapping

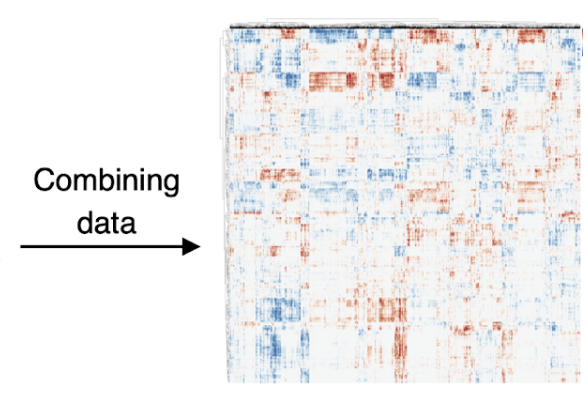

995 Fig. S8: Integration of plant and bacterial transcriptomes Schematic diagram 996 showing a bootstrapping approach to evaluate correlations between individual 997 plant and bacterial genes. To obtain robust correlation scores, Pearson's 998 correlation coefficients were calculated using all the combinations of eight strains 999 as well as using all the nine strains. Among these 8-strain and 9-strain datasets, 1000 the weakest correlation coefficient value was used for each combination of a 1001 bacterial OG and a plant gene ("Combining data"). 

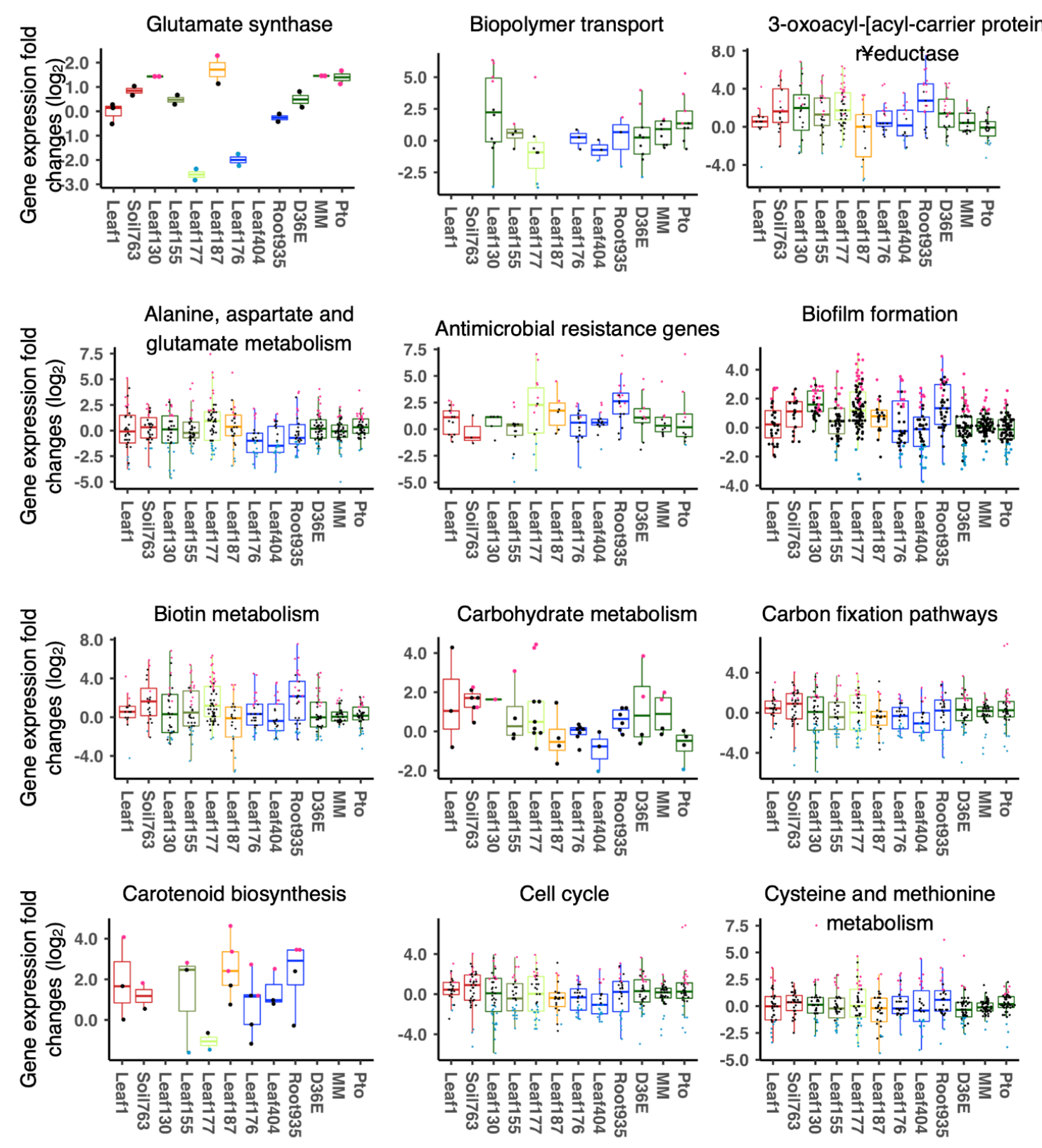

Cell cycle
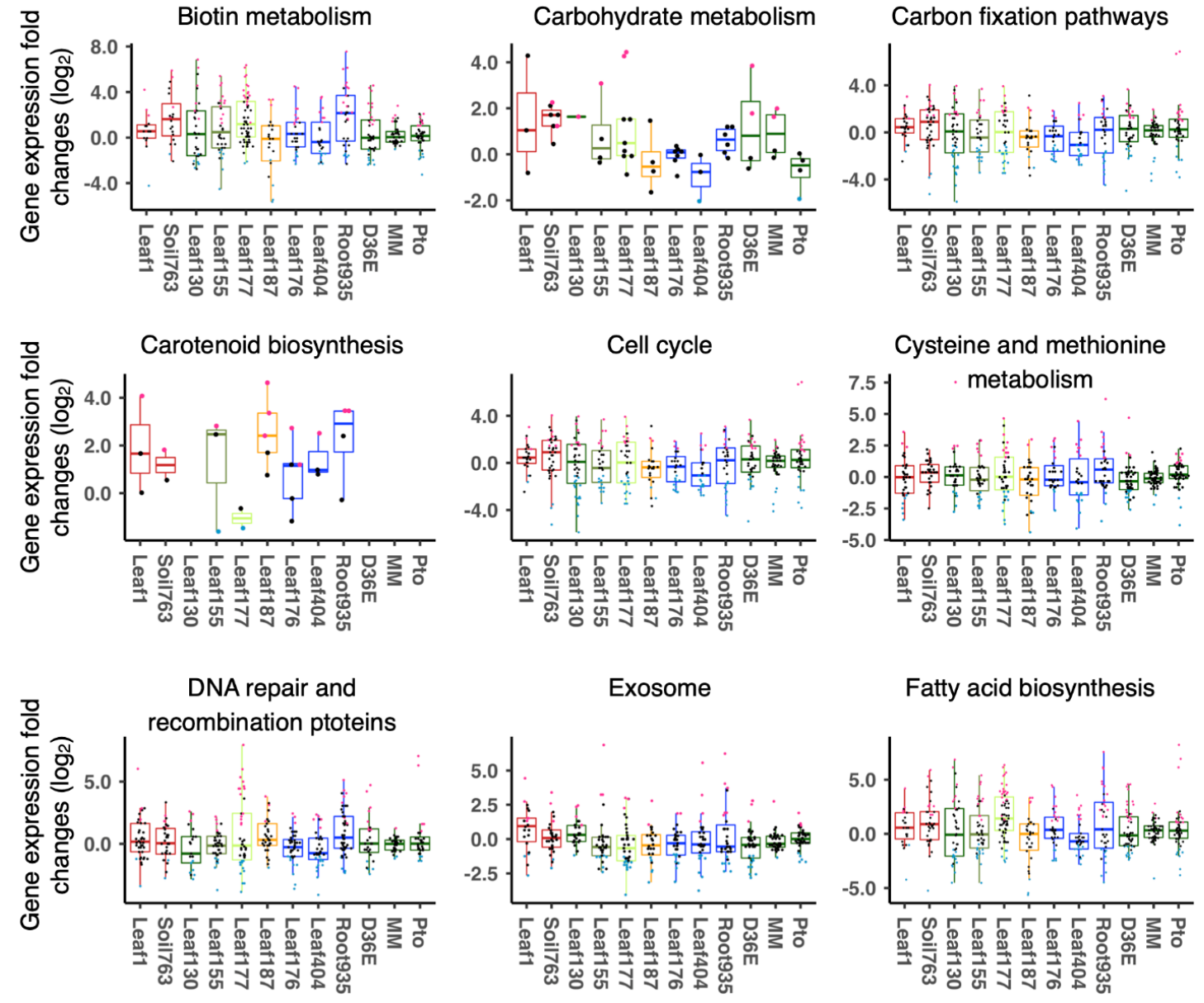

Fatty acid biosynthesis

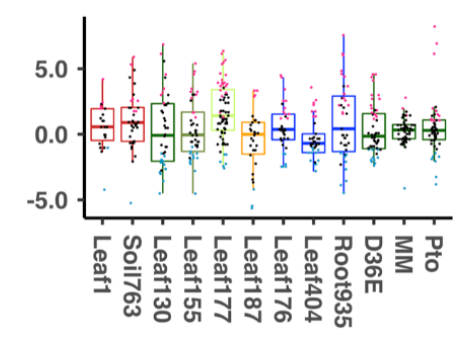

Nobori et al. | Co-transcriptomics of plants and microbiota 
bioRxiv preprint doi: https://doi.org/10.1101/2021.04.25.440543; this version posted April 26, 2021. The copyright holder for this preprint (which

was not certified by peer review) is the author/funder, who has granted bioRxiv a license to display the preprint in perpetuity. It is made available under aCC-BY-NC-ND 4.0 International license.

1002 Fig. S9: Expression of commensals genes related to various physiological 1003 processes in planta Expression fold changes (in planta vs. in vitro) of genes 1004 related to various functions. Results are shown as box plots with boxes 1005 displaying the 25th-75th percentiles, the centerline indicating the median, 1006 whiskers extending to the minimum, and maximum values no further than 1.5 1007 inter-quartile range. Box color indicates the taxonomic affiliation (phylum/class 1008 level) of each strain. All individual data points (genes) are overlaid with colors for 1009 DEGs (red: upregulated, blue: downregulated, black: non-DEG). 

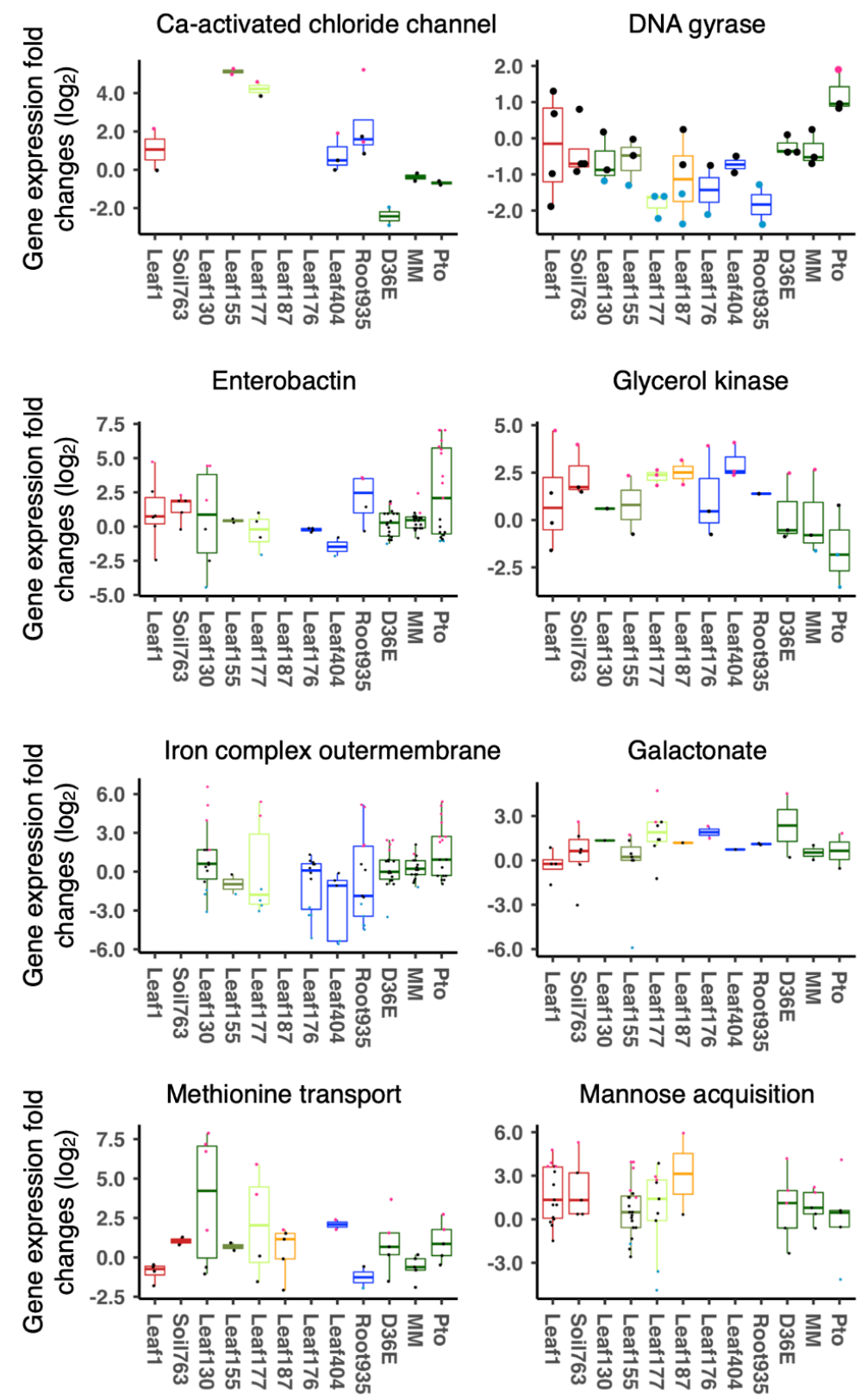

Putative tricarboxylic transport
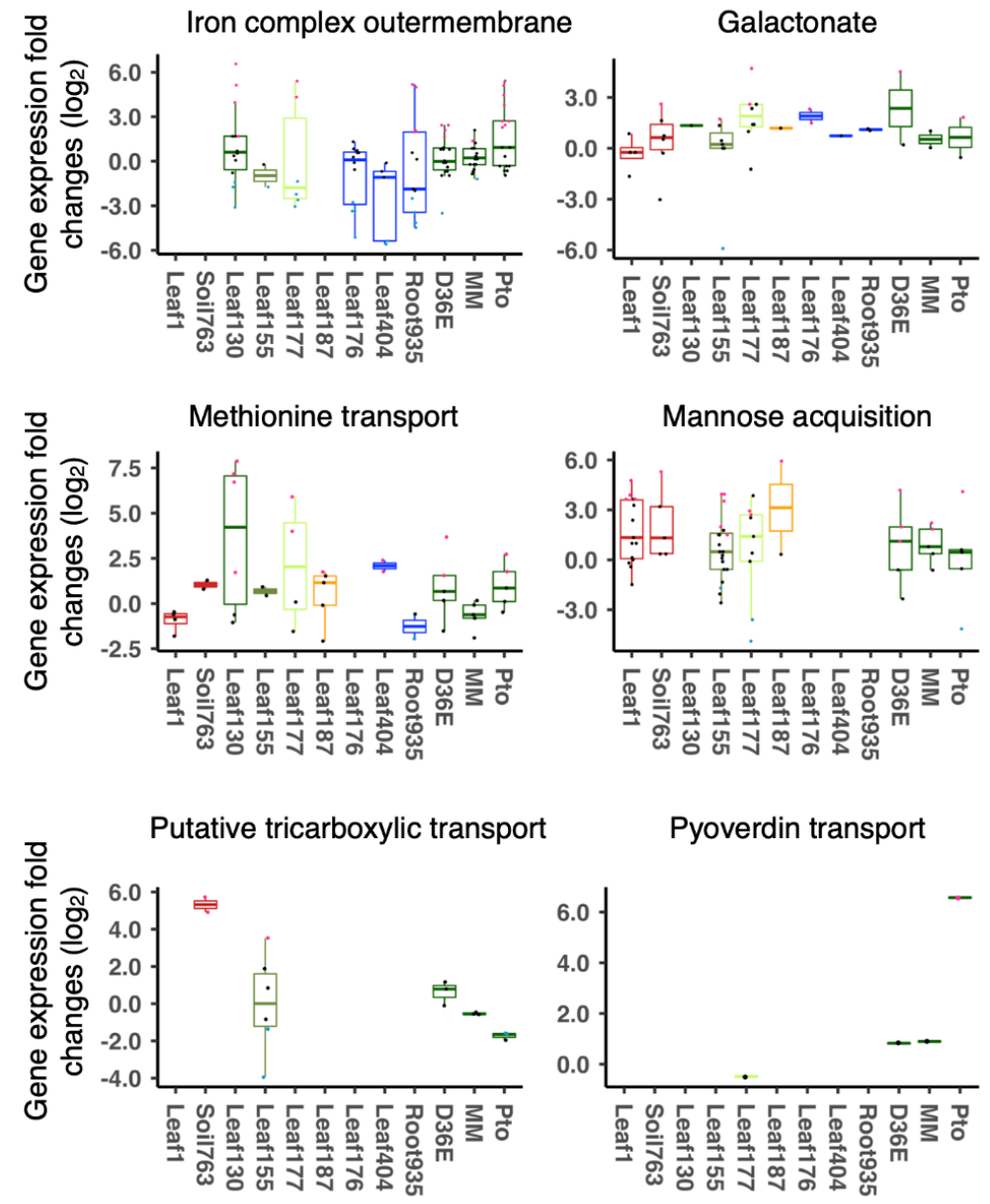

Mannose acquisition

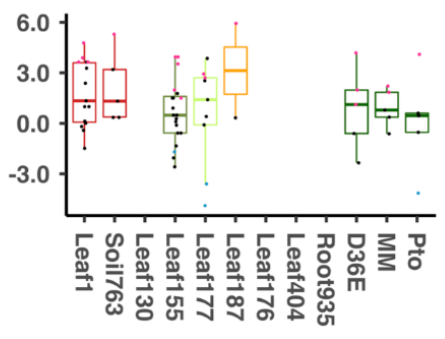

Pyoverdin transport

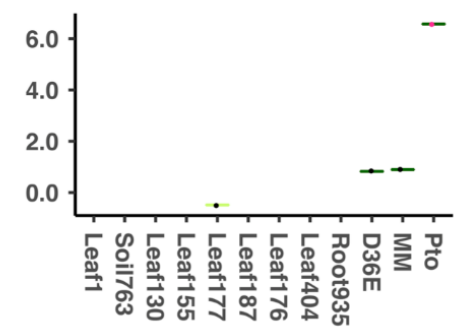

DNA-directed RNA polymerase

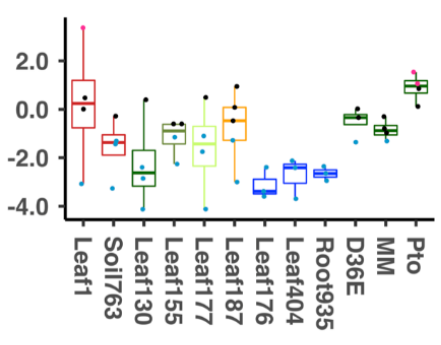

Phosphate transport

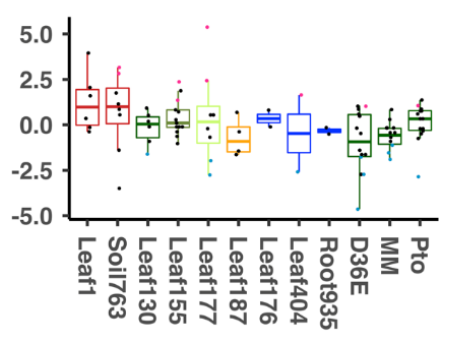

Polysaccharide biosynthesis

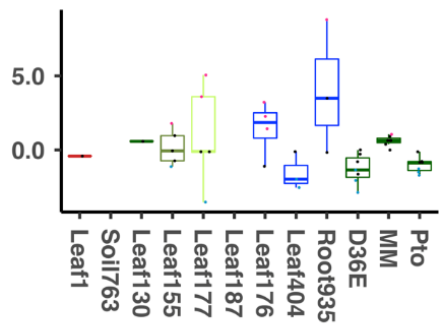

Methylmethionine transporter

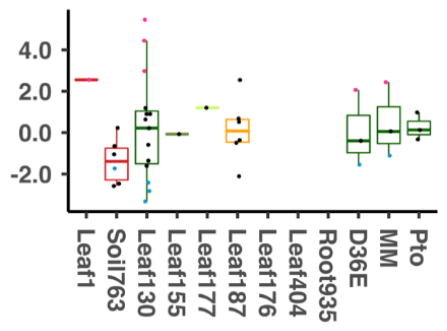

Alginate production

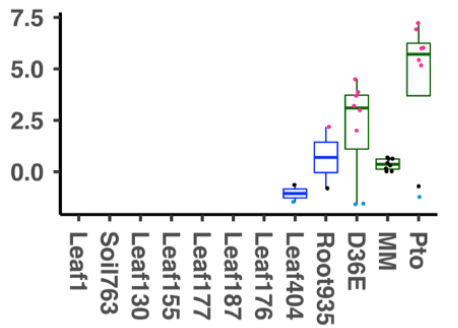

1011 Fig. S10: Expression of commensal genes related to various physiological 1012 processes in planta Expression fold changes (in planta vs. in vitro) of genes 1013 related to various functions. Results are shown as box plots with boxes 
bioRxiv preprint doi: https://doi.org/10.1101/2021.04.25.440543; this version posted April 26, 2021. The copyright holder for this preprint (which

was not certified by peer review) is the author/funder, who has granted bioRxiv a license to display the preprint in perpetuity. It is made available under aCC-BY-NC-ND 4.0 International license.

1014 displaying the 25th-75th percentiles, the centerline indicating the median, 1015 whiskers extending to the minimum, and maximum values no further than 1.5 1016 inter-quartile range. Box color indicates the taxonomic affiliation (phylum/class 1017 level) of each strain. All individual data points (genes) are overlaid with colors for 1018 DEGs (red: upregulated, blue: downregulated, black: non-DEG). 

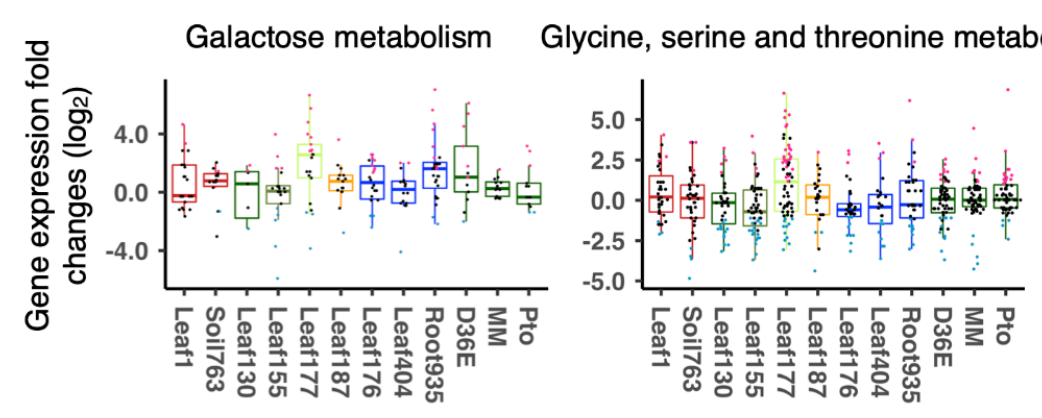

Glycolysis

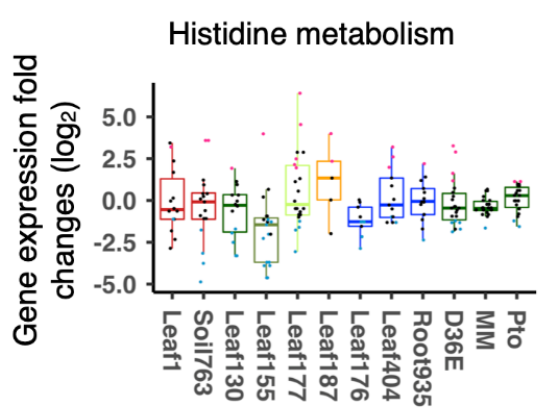

Lipopolysaccharide biosynthesis
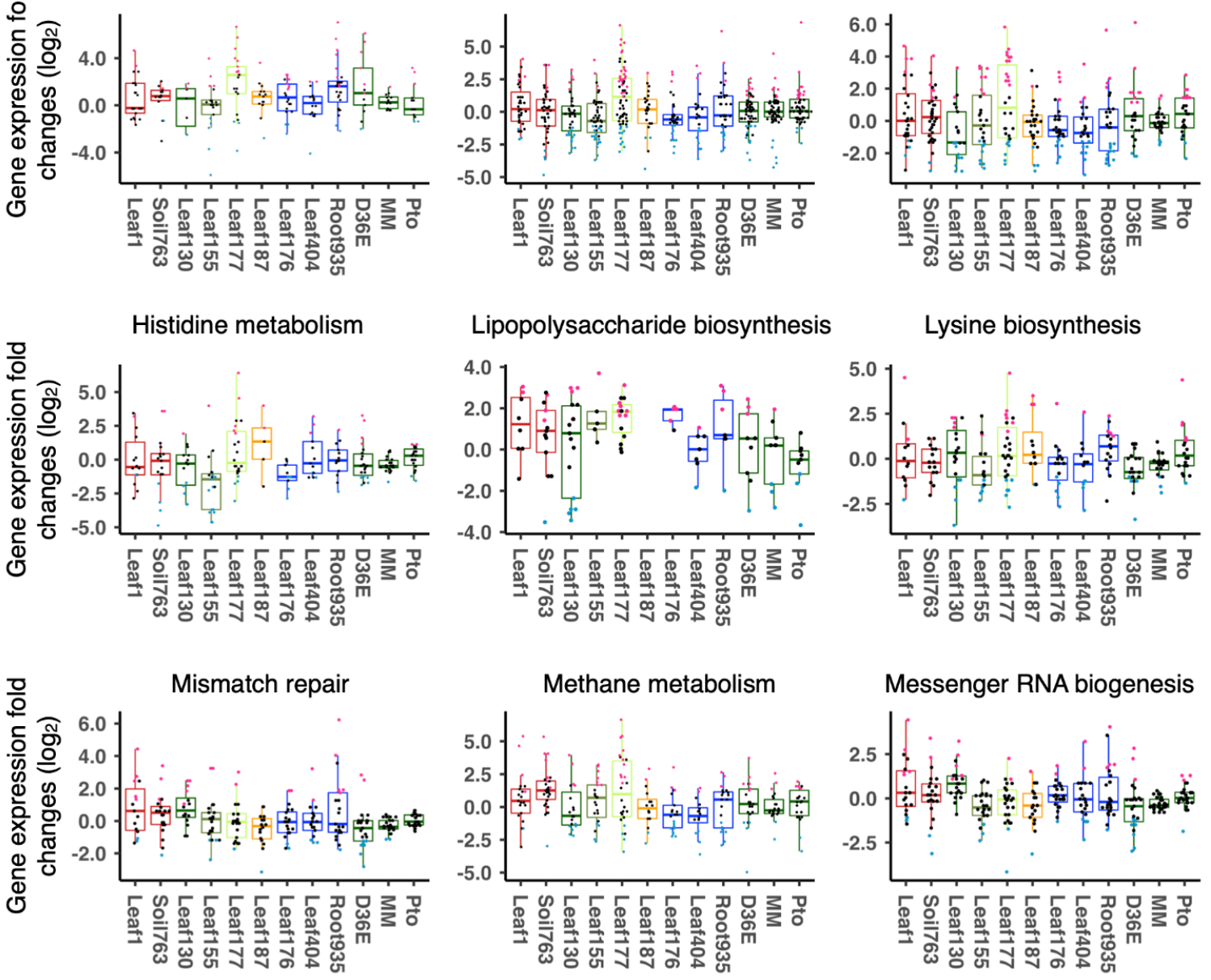
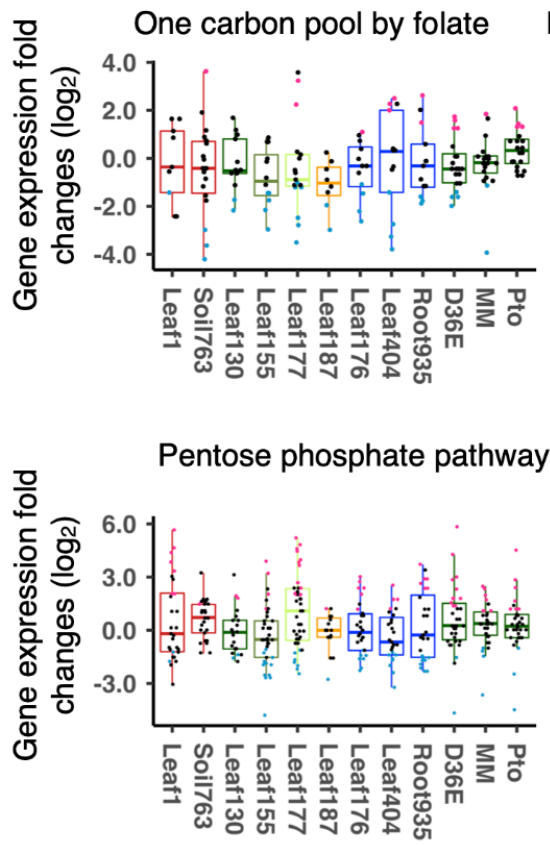

Nicotinate and nicotinamide metabolism Monobactam biosynthesis

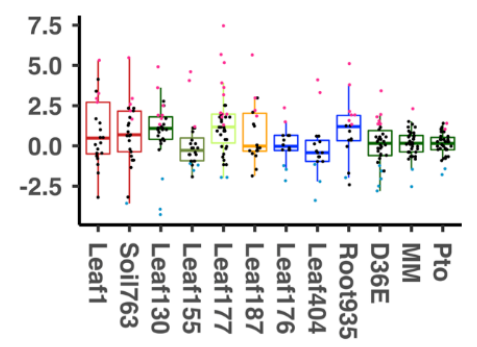

Peptidases

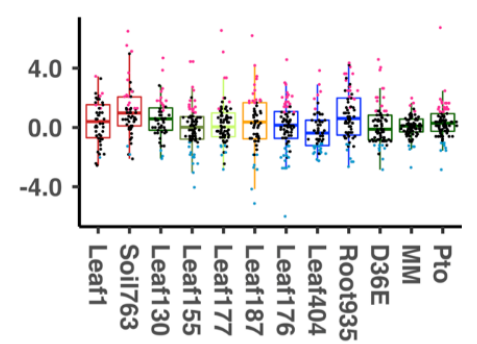

Messenger RNA biogenesis

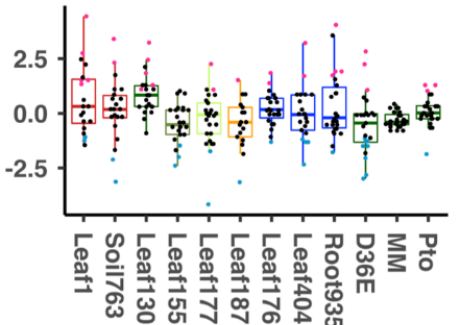

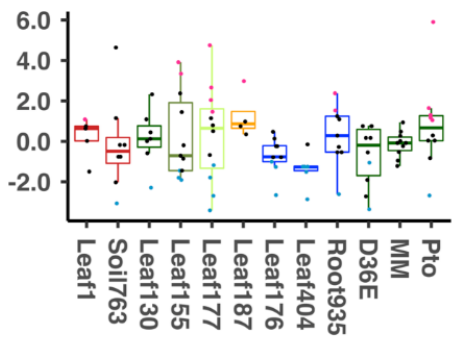

Peptidoglycan biosynthesis

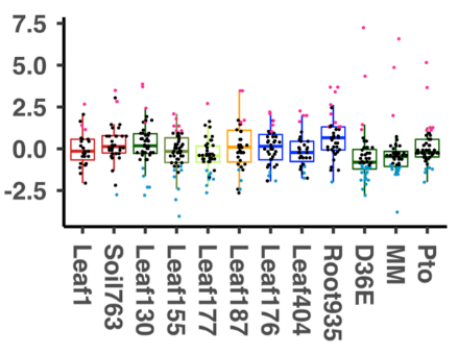


bioRxiv preprint doi: https://doi.org/10.1101/2021.04.25.440543; this version posted April 26, 2021. The copyright holder for this preprint (which

was not certified by peer review) is the author/funder, who has granted bioRxiv a license to display the preprint in perpetuity. It is made available under aCC-BY-NC-ND 4.0 International license.

1019 Fig. S11: Expression of commensal genes related to various physiological 1020 processes in planta Expression fold changes (in planta vs. in vitro) of genes 1021 related to various functions. Results are shown as box plots with boxes 1022 displaying the 25th-75th percentiles, the centerline indicating the median, 1023 whiskers extending to the minimum, and maximum values no further than 1.5 1024 inter-quartile range. Box color indicates the taxonomic affiliation (phylum/class 1025 level) of each strain. All individual data points (genes) are overlaid with colors for 1026 DEGs (red: upregulated, blue: downregulated, black: non-DEG). 

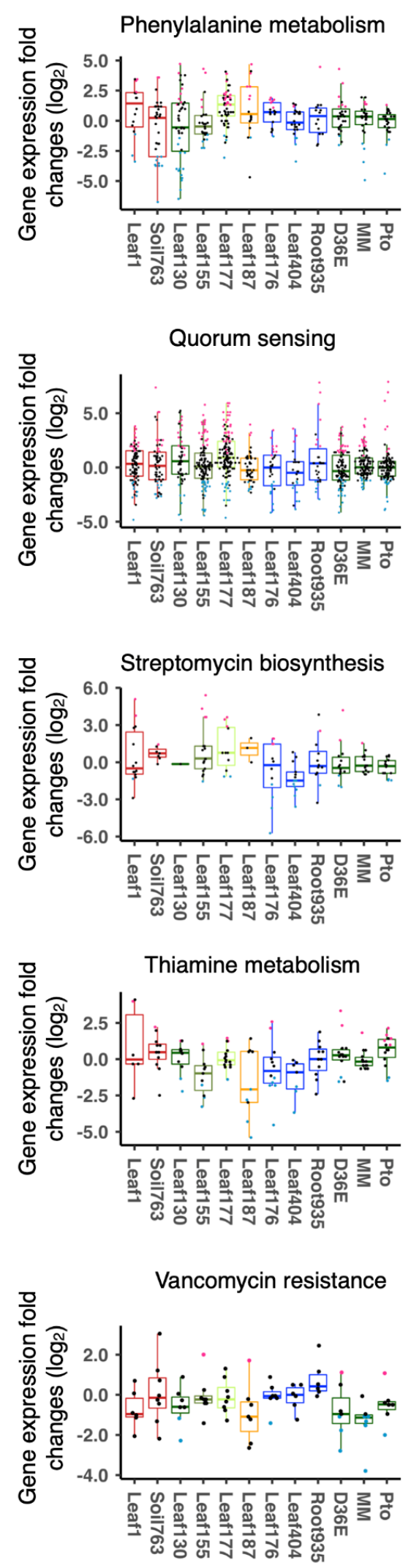

Purine metabolism

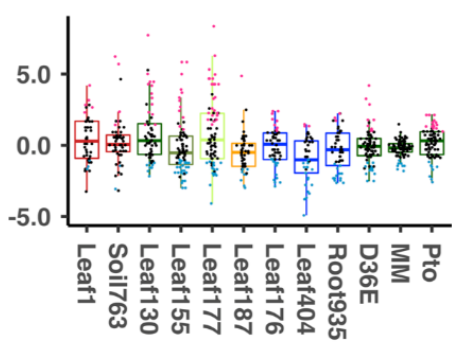

Pyruvate metabolism

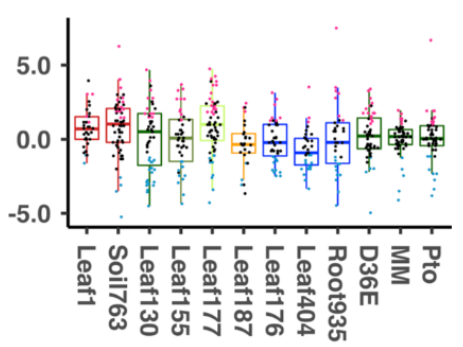

Starch and sucrose metabolism

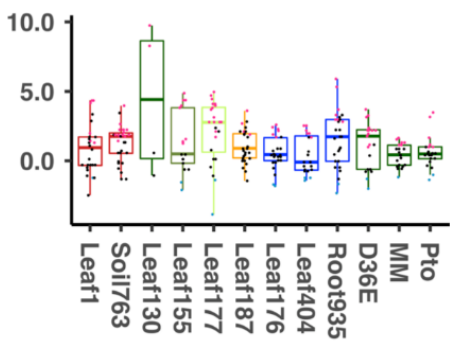

Synthesis and degradation

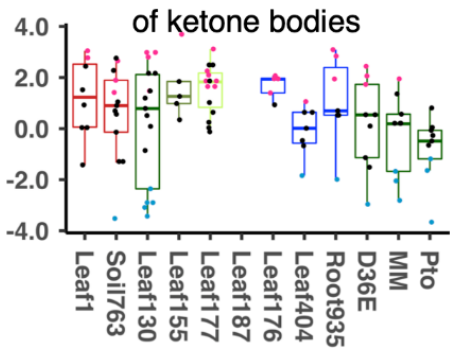

Valine, leucine and isoleucine degradation

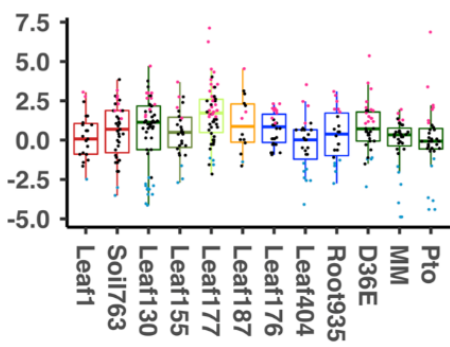

Porphyrin and chlorophyll metabolism

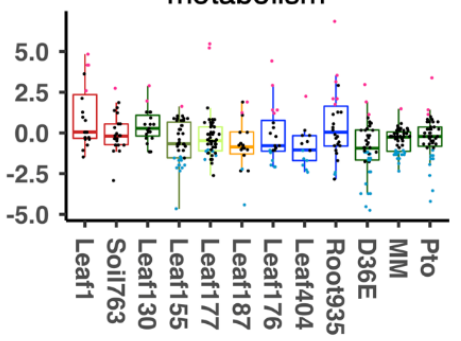

Pyrimidine metabolism

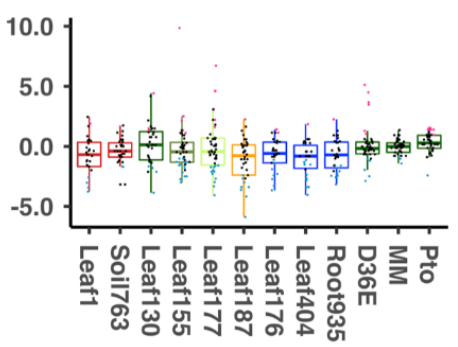

RNA degradation

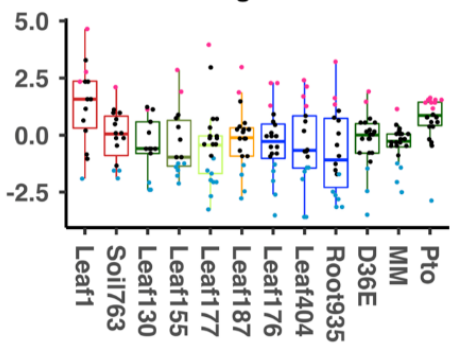

Transcription factors

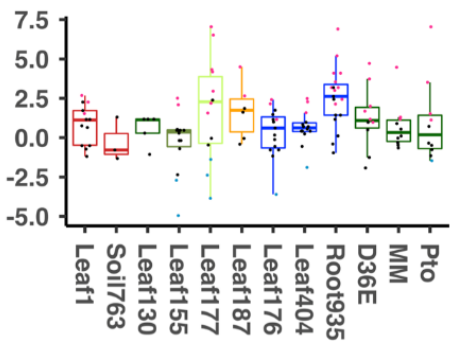

Tryptophan metabolism

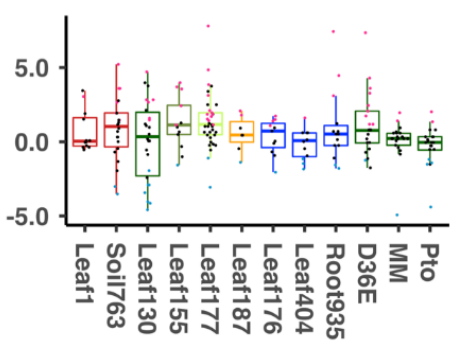


bioRxiv preprint doi: https://doi.org/10.1101/2021.04.25.440543; this version posted April 26, 2021. The copyright holder for this preprint (which

was not certified by peer review) is the author/funder, who has granted bioRxiv a license to display the preprint in perpetuity. It is made available under aCC-BY-NC-ND 4.0 International license.

1027 Fig. S12: Expression of commensal genes related to various physiological 1028 processes in planta Expression fold changes (in planta vs. in vitro) of genes 1029 related to various functions. Results are shown as box plots with boxes 1030 displaying the 25th-75th percentiles, the centerline indicating the median, 1031 whiskers extending to the minimum, and maximum values no further than 1.5 1032 inter-quartile range. Box color indicates the taxonomic affiliation (phylum/class 1033 level) of each strain. All individual data points (genes) are overlaid with colors for 1034 DEGs (red: upregulated, blue: downregulated, black: non-DEG). 